\title{
Iterative Matrix Decomposition Aided Block Diagonalization for mm-Wave Multiuser MIMO Systems
}

\author{
Rakshith Rajashekar and L. Hanzo, Fellow, IEEE
}

\begin{abstract}
Considering the dearth for spectrum in the congested microwave band, the next generation of cellular communication systems is envisaged to incorporate part of the millimeter wave (mm-wave) band. Hence recently, there has been a significant interest in beamforming aided mm-wave systems. We consider a downlink multiuser mm-wave system employing a large number of antennas combined with a fewer radio frequency (RF) chains both at the base station (BS) and at each of the user equipment (UE). The BS and each of the UE is assumed to have a hybrid beamforming architecture, where a set of analog phase shifters is followed by digital precoding/combining blocks. In this paper, 1) we propose an iterative matrix decomposition based hybrid beamforming (IMD-HBF) scheme for a singleuser scenario, which accurately approximates the unconstrained beamforming solution, 2) we show that the knowledge of the angle of departure (AoD) of the various channel paths is sufficient for the block diagonalization (BD) of the downlink mm-wave channel and hence for achieving interference free channels for each of the UEs, 3) we propose a novel subspace projection based AoD aided BD (SP-AoD-BD) that achieves significantly better performance than the conventional $\mathrm{BD}$, while still only requiring the knowledge of the $\mathrm{AoD}$ of various channel paths, 4) we use IMD-HBF in order to employ SP-AoD-BD in the hybrid beamforming architecture and study its performance with respect to the unconstrained system. We demonstrate using simulation results that the proposed IMD-HBF gives the same spectral efficiency as that of the unconstrained system in the single user scenario. Furthermore, we study the achievable sum rate of the users, when employing SP-AoD-BD with the aid of IMD-HBF and show that the loss in the performance with respect to the unconstrained system as well as the existing schemes is negligible, provided that the number of users is not excessive.
\end{abstract}

Index Terms-Block diagonalization, mm-wave communication, Hybrid beamforming, precoding and combining.

\section{INTRODUCTION}

The increased demand for higher data rates in 5G are envisaged to be met by utilizing the large unlicensed spectral resources of millimeter wave (mm-wave) frequencies [1][3]. However, operating at mm-wave frequencies comes with several hurdles that have to be overcome, which include the severe path loss due to the high operating frequency, limited scattering resulting in both spatially and temporally

R. Rajashekar and L. Hanzo are with the School of ECS, University of Southampton, UK (e-mail: rmr1u14@soton.ac.uk, lh@ecs.soton.ac.uk).

The financial support of the EPSRC projects EP/Noo4558/1 and EP/L018659/1, as well as of the European Research Council's Advanced Fellow Grant under the Beam-Me-Up project and of the Royal Society's Wolfson Research Merit Award is gratefully acknowledged. All data supporting this study are openly available from the University of Southampton repository at http://eprints.soton.ac.uk/id/eprint/405410 under doi:10.5258/SOTON/405410 sparse channels [4], [5], the high implementation complexity of the associated multi-carrier system etc. The issue of path loss and channel sparsity can be overcome by employing beamforming $(\mathrm{BF})$ at both transmitter and receiver, which allows us to achieve a sufficiently high signal-to-noise ratio (SNR) [6], which is imperative for reliable communication. Furthermore, operating at mm-wave frequencies has the advantage of accommodating a large number of antennas in a limited space, since the antenna dimension as well as the $\lambda / 2$-based antenna spacing will be of the order of millimeters. Since employing digital BF for a large number of antennas imposes a high system complexity, a hybrid architecture that uses analog phase shifters in conjunction with a reduced number of radio frequency (RF) chains is conceived [7]-[14], [29], [30]. Beamforming using this hybrid architecture is often referred to as hybrid beamforming (HBF). Furthermore, the antenna array structures considered in the HBF literature can be broadly classified as 1) Full-array structures (FAS), where the signals from all the receive (and transmit) antennas are phase-shifted and combined for baseband processing at the receiver (and transmitter) and 2) Array of sub-arrays (ASA), where the signals from a subset of receive (and transmit) antennas are phase shifted and combined for baseband processing at the receiver (and transmitter).

The optimal precoding/combining matrices designed for $\mathrm{HBF}$ are obtained by maximizing the system capacity over the feasible set of analog and digital BF matrices at both the transmitter and receiver [7]-[14]. Specifically, the authors of [8]-[10] propose a Basis Pursuit aided method for obtaining an analog BF matrix and a Least Squares (LS) based method for the digital BF matrix computation, $\mathrm{Ni}$ et al [11] propose a Convex Quadratic Programming based approach for obtaining an analog BF matrix and an LS based digital BF matrix computation method, [12] while Singh and Ramakrishna propose a dominant beam based selection approach for obtaining an analog BF matrix and then invoke a predefined codebook for the digital BF matrix. Furthermore, Dai et al [13] present a successive interference cancellation based approach for obtaining the analog $\mathrm{BF}$ matrix and a mean square error minimization approach for the digital BF matrix computation, Raghavan et al [14] study the advantages of directional BF by considering two-path mm-wave channels. All the existing approaches either assume the knowledge of full channel state information (CSI) or of partial CSI and obtain the optimal $\mathrm{BF}$ matrices that best approximate the dominant left and right singular vectors of the channel matrix. 
TABLE I

COMPARISON OF VARIOUS EXISTING HBF SOLUTIONS FOR MULTI-USER COMMUNICATION.

\begin{tabular}{|c|c|c|c|c|c|c|}
\hline & $\begin{array}{c}\text { BS } \\
\text { Antenna array }\end{array}$ & $\begin{array}{c}\text { BS } \\
\text { Analog BF }\end{array}$ & $\begin{array}{c}\text { BS } \\
\text { Digital BF }\end{array}$ & $\begin{array}{l}\text { BS } \\
\text { CSI }\end{array}$ & $\begin{array}{c}\text { No. of streams } \\
\text { per user }\end{array}$ & $\begin{array}{l}\text { Comments on BF } \\
\text { and CSI usage }\end{array}$ \\
\hline Le Liang et al [16] & FAS & $\begin{array}{l}\mathrm{EGT} / \\
\mathrm{PZF}\end{array}$ & $\mathrm{ZF}$ & Full and perfect & 1 & Generic \\
\hline Stirling et al [17] & ASA & $\begin{array}{c}\text { Combinatorial } \\
\text { search }\end{array}$ & MMSE/BD & Full and perfect & $N_{s} \geq 1$ & Generic \\
\hline Jinho Choi [18] & FAS & OMP & None & Imperfect estimate & 1 & $\begin{array}{l}\text { Specific to } \\
\text { mm-wave }\end{array}$ \\
\hline Ahmed et al [19] & FAS & $\begin{array}{l}\text { Combinatorial } \\
\text { search }\end{array}$ & $\mathrm{ZF}$ & $\begin{array}{l}\text { Imperfect baseband } \\
\text { channel estimate }\end{array}$ & 1 & $\begin{array}{l}\text { Specific to } \\
\text { mm-wave }\end{array}$ \\
\hline Weiheng et al [20] & FAS & EGT & $\mathrm{BD}$ & Full and perfect & $N_{s} \geq 1$ & Generic \\
\hline Bogale et al $[21] \dagger$ & FAS & OMP & $\mathrm{LS}$ & Full and perfect & $N_{s} \geq 1$ & $\begin{array}{l}\text { Specific to } \\
\text { mm-wave }\end{array}$ \\
\hline
\end{tabular}

$\dagger$ Approximates conventional full-digital BD solution.

Apart from the above methods conceived for single-user communication, recently there have been a few significant developments in $\mathrm{HBF}$ also for multi-user (MU) communication [16]-[20]. Specifically, Liang et al [16] present a phasedzero forcing (PZF) method, where the analog BF matrix of the base station (BS) is chosen to be the phase matrix of the conjugate of the composite downlink channel, while the digital precoder is chosen based on the conventional zero forcing (ZF) approach. The analog beam selection of [16] is similar to that proposed in [22]. In [17], the authors propose both single and multi-user based analog beam selection, while considering both minimum mean square error (MMSE) and ZF based solutions for digital precoding. In [18], the authors propose beam selection algorithms based on the compressive sensing approach, which rely on the orthogonal matching pursuit (OMP) algorithm [23] for finding dominant beams. In [19], the authors propose a two-stage algorithm in which the first stage obtains the analog BF matrices and the second stage obtains the digital BF matrix based on the classic zero-forcing approach. In [20], the authors propose equal gain transmission (EGT) based analog BF combined with block diagonalization (BD) based digital precoding. It is worth mentioning that most of these schemes are generic in the sense that they do not exploit the mm-wave channel characteristics and hence can be used in other channels as well. In [21], the authors consider the conventional BD solution [25] and then approximate it with the aid of an OMP based hybrid BF solution. The performance of the proposed hybrid BF solution in [21] is upper bounded by that of the full-digital version [25]. Table I compares various aspects of the above methods.

Against this background, the following are the new contributions of this paper.

1) Considering an FAS based single user communication system, we propose a low-complexity iterative matrix decomposition (IMD) that factors the dominant singular vectors of the channel into equal-gain element analog $\mathrm{BF}$ matrix and a power-constrained digital $\mathrm{BF}$ matrix. We show with the aide of simulation results that the proposed IMD based solution gives an accurate representation of the singular vectors and hence achieves nearly the same throughput as that of the unconstrained massive multiple input multiple output (MIMO) system. Furthermore, we evaluate the angular resolution of the analog phase shifters required for achieving nearly the same throughput as that attained by having infiniteresolution phase shifters.

2) It is widely recognized that operating at mm-wave frequencies is expected to impose less inter-user interference compared to systems operating at microwave frequencies, an advantage which is yet to be sufficiently exploited. Note that most of the schemes considered in Table I are generic and do not explicitly exploit the specific characteristics of the mm-wave channel. By exploiting the spatial-domain sparsity of the mm-wave channel, first we show that it is not necessary to have full CSI at the BS. Indeed, having only the knowledge of the angle of departure (AoD) of the signal paths associated with the various users is sufficient for achieving BD of the composite multi-user channel. This method of BD is termed as AoD aided BD (AoD-BD), which was first studied in [25] (Section III-C), although not in the context of mm-wave communication. Even though, the knowledge of AoD is sufficient to block-diagonalize the multi-user channel, we show that this approach is not suitable for mm-wave communication, since it would result in poor signal power owing to the channel's spatial sparsity. We propose a novel BD scheme in order to overcome this problem by ensuring that the blockdiagonalized channel of each user falls in the corresponding user signal-space, thus enhancing the user's 
signal quality. Note that this approach is fundamentally different from the existing BD schemes [24], [25], where the precoders are designed only to block-diagonalize the multi-user channel without any explicit motivation to enhance the signal power. The BD schemes in the existing mm-wave communication literature are employed in the digital domain, while the RF precoding is generally employed using beam-steering [18], [19]. Explicitly, the $\mathrm{BF}$ and $\mathrm{BD}$ are performed independently in the analog and digital domains, respectively. In contrast to this, the proposed scheme combines BD and BF into a single amalgamated operation, resulting in a novel digital BD precoder which is different from the existing BD solution [25]. The proposed scheme is termed as subspace projection based AoD aided BD (SP-AoD-BD). While the proposed scheme enjoys the benefit of partial CSI for achieving $\mathrm{BD}$, it suffers from a marginal performance loss w.r.t. the existing schemes (see Section V), when the number of users $K$ is excessive. Note that this issue can be overcome by employing a larger antenna array at the $\mathrm{BS}$, thus making the proposed solution an attractive downlink mm-wave communication technique.

3) The above SP-AoD-BD is proposed for unconstrained systems. Hence we further extend it to realistic constrained FAS based systems with the aid of the IMD based HBF described in 1) above. This scheme is referred to as the subspace projection based AoD aided BD combined with HBF (SP-BD-HBF).

The remainder of the paper is organized as follows. Section II outlines the system model that describes the BS and the UE antenna arrays as well as the mm-wave channel model considered. In Section III, we present our IMD proposed for a single user scenario. Section IV presents the proposed AoDBD and SP-AoD-BD methods. Finally, our simulation results and discussions are presented in Section V, while Section VI concludes the paper.

Notations: Uppercase and lowercase boldface letters represent matrices and vectors, respectively. The Frobenius norm of a matrix or the two-norm of a vector is represented by $\|\cdot\|$. Furthermore, $\mathcal{C N}\left(\mu, \sigma^{2}\right)$ denotes a complex Gaussian random variable with mean $\mu$ and variance $\sigma^{2}$, and the field of complex and real numbers are represented by $\mathbb{C}$ and $\mathbb{R}$, respectively. If $\mathbf{B} \in \mathbb{C}^{m \times n}$ such that $a_{i, j} e^{j b_{i, j}}$ is the polar representation of the $(i, j)^{\text {th }}$ element $\mathbf{B}_{(i, j)}$, then $\measuredangle \mathbf{B}$ represents a matrix whose $(i, j)^{\text {th }}$ element $\measuredangle \mathbf{B}_{(i, j)}=e^{j b_{i, j}}$. The $|\mathbf{B}|$ represents the determinant of the matrix $\mathbf{B}$. The $\operatorname{span}(\mathbf{B})$ represents the space spanned by the columns of $\mathbf{B}$. Furthermore, the notations of $(\cdot)^{T},(\cdot)^{H}$, and $(\cdot)^{*}$ indicate the transpose, Hermitian transpose, and complex conjugate of a matrix/vector, respectively. Furthermore, $\mathbf{C}([q: r],:)$ defines a matrix with rows $q, q+1, \ldots, r-1, r$ of $\mathbf{C}$ and $\mathbf{C}(:,[p: q])$ is a matrix with columns $p, p+1, \ldots, q-1, q$ of $\mathbf{C}$. The expected value of a random quantity $\mathbf{Y}$ is represented by $\mathbb{E}[\mathbf{Y}]$.

\section{System MODEL}

\section{A. Channel Model}

Assuming the geometric model of [8]-[11] for the narrowband mm-wave channel between the BS and each UE, we have

$$
\mathbf{H}_{j}=\sqrt{N_{t} N_{r}} \sum_{i=1}^{L_{j}} \beta_{i}^{(j)} \mathbf{e}_{r}\left(\theta_{i}^{(j)}\right) \mathbf{e}_{t}^{H}\left(\phi_{i}^{(j)}\right), 1 \leq j \leq K,
$$

where $L_{j}$ is the number of channel paths between the BS and the $j^{\text {th }}$ user, $\beta_{i}^{(j)} \sim \mathcal{C N}(0,1)$ is the gain of the $i^{\text {th }}$ path of the $j^{\text {th }}$ user's channel, $\left(\theta_{i}^{(j)}, \phi_{i}^{(j)}\right)$ represent the angle of arrival (AoA) and angle of departure (AoD) of the $i^{\text {th }}$ path of the $j^{\text {th }}$ user, $\mathbf{e}_{r}$ and $\mathbf{e}_{t}$ represent the spatial receive and transmit signatures of a uniform linear array (ULA), respectively, which are given by

$$
\begin{aligned}
& \mathbf{e}_{r}(\theta)=\frac{1}{\sqrt{N_{r}}}\left[1, e^{j \frac{2 \pi}{\lambda} d \cos \theta}, \ldots, e^{j \frac{2 \pi}{\lambda} d\left(N_{r}-1\right) \cos \theta}\right]^{T}, \\
& \mathbf{e}_{t}(\phi)=\frac{1}{\sqrt{N_{t}}}\left[1, e^{j \frac{2 \pi}{\lambda} d \cos \phi}, \ldots, e^{j \frac{2 \pi}{\lambda} d\left(N_{t}-1\right) \cos \phi}\right]^{T},
\end{aligned}
$$

where $d$ is the separation between the antenna elements and $\lambda$ is the carrier's wavelength. We assume that $L_{j}=L$, $\left(\theta_{i}^{(j)}, \phi_{i}^{(j)}\right) \in[0,2 \pi]$ for $1 \leq j \leq K$. Equation (1) can be expressed in a compact form as

$$
\mathbf{H}_{j}=\mathbf{E}_{r}^{(j)} \mathbf{D}^{(j)} \mathbf{E}_{t}^{(j)}{ }^{H}
$$

where

$$
\begin{aligned}
& \mathbf{E}_{r}^{(j)}=\left[\mathbf{e}_{r}\left(\theta_{1}^{(j)}\right), \mathbf{e}_{r}\left(\theta_{2}^{(j)}\right), \ldots, \mathbf{e}_{r}\left(\theta_{L}^{(j)}\right)\right], \\
& \mathbf{E}_{t}^{(j)}=\left[\mathbf{e}_{t}\left(\phi_{1}^{(j)}\right), \mathbf{e}_{t}\left(\phi_{2}^{(j)}\right), \ldots, \mathbf{e}_{t}\left(\phi_{L}^{(j)}\right)\right],
\end{aligned}
$$

and $\mathbf{D}^{(j)}$ is a diagonal matrix whose $k^{\text {th }}$ diagonal entry is given by $\mathbf{D}_{(k, k)}^{(j)}=\sqrt{N_{t} N_{r}} \beta_{k}^{(j)}$. Note that the results presented in this paper also hold for other antenna array structures as well, such as uniform planar arrays or uniform circular arrays.

\section{B. Unconstrained System}

Assuming that both the BS and the UEs have $N_{t}$ and $N_{r}$ RF chains and employ complete digital processing, the signal received at the $j^{\text {th }} \mathrm{UE}$ is given by

$$
\mathbf{y}_{j}=\mathbf{W}_{j}^{H} \mathbf{H}_{j} \mathbf{F} \mathbf{x}+\mathbf{W}_{j}^{H} \mathbf{n}_{j} \in \mathbb{C}^{N_{s}},
$$

where $\mathbf{W}_{j} \in \mathbb{C}^{N_{r} \times N_{s}}$ is the receiver's combining matrix of the $j^{\text {th }}$ user, $\mathbf{F} \in \mathbb{C}^{N_{t} \times K N_{s}}$ is the transmit precoding matrix, $\mathbf{x}=\left[\mathbf{x}_{1}^{T}, \mathbf{x}_{2}^{T}, \ldots, \mathbf{x}_{K}^{T}\right]^{T} \in \mathbb{C}^{K N_{s}}$ is the transmit vector in which the $j^{\text {th }}$ user's symbols are represented by $\mathbf{x}_{j} \in \mathbb{C}^{N_{s}}$ such that $\mathbb{E}\left[\mathbf{x x}^{H}\right]=\frac{P}{K N_{s}} \mathbf{I}_{K N_{s}}$, where $P$ is total transmit power at the BS and $\mathbf{n}_{j} \in \mathbb{C}^{N_{r}}$ is the additive white Gaussian noise vector whose elements are from $\mathcal{C N}(0,1)$. Furthermore, the precoding matrix is normalized to satisfy $\|\mathbf{F}\|^{2}=K N_{s}$ and the combining matrix of each user is normalized to satisfy $\left\|\mathbf{W}_{j}\right\|^{2}=N_{s}$. We use this system for benchmarking the performance of the various methods proposed that rely on constrained precoding and combining matrices.

\section{Constrained FAS based System}

Since having $N_{t}$ and $N_{r}$ RF chains imposes a high cost and complexity, HBF has gained popularity which uses fewer $\mathrm{RF}$ chains in conjunction with analog phase shifters [8]. Let 
us consider a MU-MIMO system, where the BS is assumed to have $N_{t}$ antennas and $M_{t} \mathrm{RF}$ chains, whilst each of the $K$ users is assumed to have $N_{r}$ antennas and $M_{r}$ RF chains. At the BS, the signal from each RF chain is appropriately phase shifted before being fed to each of the antenna elements. By contrast, at the UE, the signal received at each of the antenna elements is appropriately phase shifted before being fed to each RF chain. In this paper, we consider only downlink communication, where the BS acts as the transmitter while the UEs act as the receivers. Furthermore, the number of downlink data streams sent to each user is assumed to be $N_{s}=M_{r}$. The signal received by the $j^{\text {th }}$ user is given by

$$
\mathbf{y}_{j}=\mathbf{G}_{j}^{H} \boldsymbol{\Phi}_{j}^{H} \mathbf{H}_{j} \boldsymbol{\Theta} \mathbf{C x}+\mathbf{G}_{j}^{H} \boldsymbol{\Phi}_{j}^{H} \mathbf{n}_{j} \quad \in \mathbb{C}^{N_{s}},
$$

where $\boldsymbol{\Phi}_{j} \in \mathbb{C}^{N_{r} \times N_{s}}$ and $\mathbf{G}_{j} \in \mathbb{C}^{N_{s} \times N_{s}}$ are the analog and digital combining matrices of the $j^{\text {th }}$ user, respectively, $\boldsymbol{\Theta} \in$ $\mathbb{C}^{N_{t} \times K N_{s}}$ and $\mathbf{C} \in \mathbb{C}^{K N_{s} \times K N_{s}}$ are the analog and digital precoding matrices, respectively. Furthermore, the analog and digital precoding matrices are normalized to satisfy $\|\mathbf{\Theta C}\|^{2}=$ $K N_{s}$, while the analog and digital combining matrices of each user are normalized to satisfy $\left\|\boldsymbol{\Phi}_{j} \mathbf{G}_{j}\right\|^{2}=N_{s}$.

\section{Proposed Iterative Matrix Decomposition for $\mathrm{HBF}$}

Considering the single user scenario $(j=1)$, the optimal precoding and combining matrices for the unconstrained system are the right and left singular vectors associated with the $N_{s}$ dominant singular values of the channel. Several methods are available in the existing literature that approximate these singular vectors [7]-[13] by the product of the analog and digital precoding/combining matrices. Most of these methods use matrices constituted by the beam steering vectors or equal gain elements in order to obtain analog precoding/combining matrices. In this section, we present a simple iterative method conceived for obtaining the analog and digital precoding/combining matrices that are close approximations of the singular vectors that are optimal for unconstrained systems. We assume that the transmitter and receiver have perfect knowledge of the right and left singular vectors of the channel, which can be acquired with the aid of channel sounding (refer Section III-A [9]).

Algorithm 1 presented below gives the details of the proposed iterative method of obtaining the precoding/combining matrices for HBF.

The physical interpretation of the above algorithm is as follows. In Step 1 of the Algorithm 1, the equal-gain element precoding/combining matrices are obtained. In Step 2, the digital precoding/combining matrices are generated that best approximate the unconstrained solution in the LS sense. In Step 3, the residual information in the unconstrained solution is obtained, which is approximated in the next iteration. Step 4 gives the precoding and combining matrices for the constrained FAS based system. We term this approach as the IMD based HBF (IMD-HBF).

\section{A. Convergence of IMD-HBF:}

The main idea used in the IMD algorithm is to alternatively project the unconstrained solution into the feasible spaces of

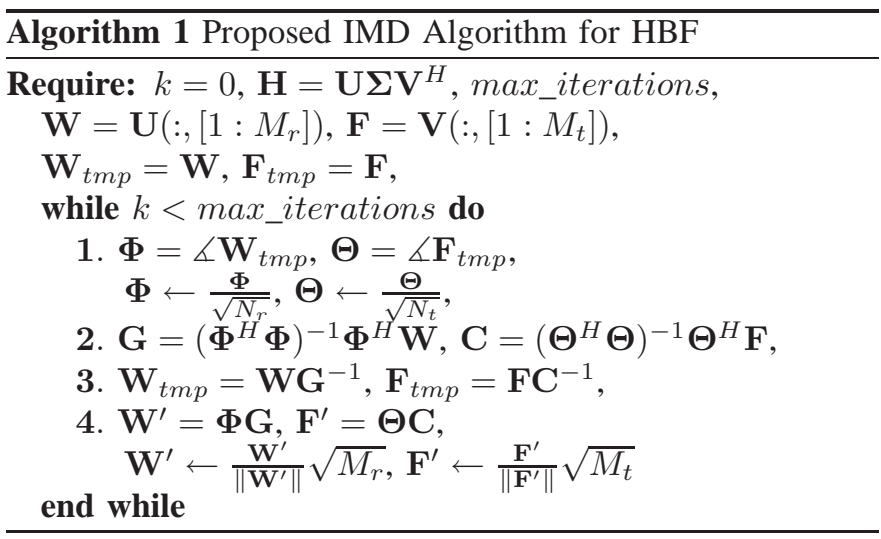

the analog and digital BF matrices, so that the residual error monotonically reduces every iteration. Fig. 1 gives a pictorial depiction of the IMD iterations. Before proceeding further, let us introduce the following definition.

Definition 1: Let $\mathbf{A}$ and B selected from $\mathbb{C}^{m \times n}$, with $m \gg$ $n$. The subspaces $\operatorname{span}(\mathbf{A})$ and $\operatorname{span}(\mathbf{B})$ are said to be nonintersecting or parallel, if $\mathbf{C}=[\mathbf{A} \mathbf{B}]$ has rank of $2 n$. In other words, $\operatorname{span}(\mathbf{A}) \cap \operatorname{span}(\mathbf{B})=\phi$.

Assuming that the $\mathrm{HBF}$ precoding and combining matrices in the $k^{\text {th }}$ iteration are $\mathbf{F}_{k}^{\prime}=\boldsymbol{\Theta}_{k} \mathbf{C}_{k}$ and $\mathbf{W}_{k}^{\prime}=\boldsymbol{\Phi}_{k} \mathbf{G}_{k}$, the residual error is given by

$$
\begin{aligned}
\mathbf{F}-\mathbf{F}_{k}^{\prime} & =\mathbf{F}-\boldsymbol{\Theta}_{k} \mathbf{C}_{k}=\boldsymbol{\Delta}_{k}, \\
\mathbf{W}-\mathbf{W}_{k}^{\prime} & =\mathbf{W}-\boldsymbol{\Phi}_{k} \mathbf{G}_{k}=\boldsymbol{\Gamma}_{k} .
\end{aligned}
$$

We use the following terms in order to quantify the residual error as a function of the number of iterations:

$$
\begin{aligned}
\left\|\boldsymbol{\Delta}_{k}\right\|^{2} & =\left\|\mathbf{F}-\mathbf{F}_{k}^{\prime}\right\|^{2}=\left\|\mathbf{F}-\boldsymbol{\Theta}_{k} \mathbf{C}_{k}\right\|^{2}, \\
\left\|\boldsymbol{\Gamma}_{k}\right\|^{2} & =\left\|\mathbf{W}-\mathbf{W}_{k}^{\prime}\right\|^{2}=\left\|\mathbf{W}-\mathbf{\Phi}_{k} \mathbf{G}_{k}\right\|^{2},
\end{aligned}
$$

where (9) represents the error in $\mathbf{F}_{k}^{\prime}$ and (10) gives the error in $\mathbf{W}_{k}^{\prime}$. The matrices $\mathbf{F}_{t m p}$ and $\mathbf{W}_{t m p}$ used in the next iteration are given by

$$
\begin{aligned}
\mathbf{F C}_{k}^{-1} & =\boldsymbol{\Theta}_{k}+\boldsymbol{\Delta}_{k} \mathbf{C}_{k}^{-1}, \\
\mathbf{W G}_{k}^{-1} & =\boldsymbol{\Phi}_{k}+\boldsymbol{\Gamma}_{k} \mathbf{G}_{k}^{-1},
\end{aligned}
$$

where the terms $\boldsymbol{\Delta}_{k} \mathbf{C}_{k}^{-1}$ and $\boldsymbol{\Gamma}_{k} \mathbf{G}_{k}^{-1}$ act as the correction factors for the analog precoding and combining matrices, since they belong to the orthogonal space of $\boldsymbol{\Theta}_{k}$ and $\boldsymbol{\Phi}_{k}$, respectively. This can be readily verified as follows:

$$
\begin{aligned}
\boldsymbol{\Theta}_{k}^{H} \boldsymbol{\Delta}_{k} & =\boldsymbol{\Theta}_{k}^{H}\left(\mathbf{F}-\boldsymbol{\Theta}_{k} \mathbf{C}_{k}\right), \\
& =\Theta_{k}^{H} \mathbf{F}-\boldsymbol{\Theta}_{k}^{H} \mathbf{\Theta}_{k} \mathbf{C}_{k}, \\
& =\Theta_{k}^{H} \mathbf{F}-\boldsymbol{\Theta}_{k}^{H} \boldsymbol{\Theta}_{k}\left(\boldsymbol{\Theta}_{k}^{H} \boldsymbol{\Theta}_{k}\right)^{-1} \boldsymbol{\Theta}_{k}^{H} \mathbf{F}, \\
& =\mathbf{O}
\end{aligned}
$$

Similarly, it can be shown that $\boldsymbol{\Phi}_{k}^{H} \boldsymbol{\Gamma}_{k}=\mathbf{O}$. In the next iteration, we have $\boldsymbol{\Theta}_{k+1}=\measuredangle\left(\mathbf{F C}_{k}^{-1}\right) / \sqrt{N_{t}}$ and $\boldsymbol{\Phi}_{k+1}=$ 
$\measuredangle\left(\mathbf{W G}_{k}^{-1}\right) / \sqrt{N_{r}}$, which satisfy

$$
\begin{aligned}
\boldsymbol{\Theta}_{k+1} & =\underset{|\boldsymbol{\Theta}(i, j)|=1 / \sqrt{N_{t}}}{\arg \min }\left\|\mathbf{F} \mathbf{C}_{k}^{-1}-\boldsymbol{\Theta}\right\|^{2}, \\
\boldsymbol{\Phi}_{k+1} & =\underset{|\boldsymbol{\Phi}(i, j)|=1 / \sqrt{N_{r}}}{\arg \min }\left\|\mathbf{W} \mathbf{G}_{k}^{-1}-\boldsymbol{\Phi}\right\|^{2} .
\end{aligned}
$$

Please refer to Appendix A for an explicit proof of (17) and (18). From (17) and (18), we have

$$
\begin{gathered}
\left\|\mathbf{F C}_{k}^{-1}-\boldsymbol{\Theta}_{k}\right\|^{2}>\left\|\mathbf{F C}_{k}^{-1}-\boldsymbol{\Theta}_{k+1}\right\|^{2}, \\
\left\|\mathbf{W G}_{k}^{-1}-\boldsymbol{\Phi}_{k}\right\|^{2}>\left\|\mathbf{W} \mathbf{G}_{k}^{-1}-\boldsymbol{\Phi}_{k+1}\right\|^{2} .
\end{gathered}
$$

Note that $\mathbf{F}, \boldsymbol{\Theta}_{k}$, and $\boldsymbol{\Theta}_{k+1}$ form a set of mutually nonintersecting subspaces ${ }^{1}$ as depicted in Iteration 2 of Fig. 2, which follows from the following facts:

1) $\boldsymbol{\Delta}_{k}$ is orthogonal to $\boldsymbol{\Theta}_{k}(16)$;

2) $\mathbf{F C}_{k}^{-1} \in \operatorname{span}(\mathbf{F})$ is obtained by translating $\boldsymbol{\Theta}_{k}$ by $\boldsymbol{\Delta}_{k} \mathbf{C}_{k}^{-1}$ (11);

3) $\boldsymbol{\Theta}_{k+1}$ is not in the space spanned by either $\mathbf{F}$ or $\boldsymbol{\Theta}_{k}$ (19).

In Fig. 2, the circle represents the set of equal-gain matrices given by

$$
\left\{\mathbf{A} \in \mathbb{C}^{N_{t} \times M_{t}}|| \mathbf{A}(i, j) \mid=1 / \sqrt{N_{t}}\right\} .
$$

From (19), it is evident that $\mathbf{F C}_{k}^{-1} \in \operatorname{span}(\mathbf{F})$ is closer to $\boldsymbol{\Theta}_{k+1}$ than to $\boldsymbol{\Theta}_{k}$. Considering the fact that $\mathbf{F}, \boldsymbol{\Theta}_{k}$, and $\boldsymbol{\Theta}_{k+1}$ are non-intersecting, it is reasonable to expect that

$$
\left\|\mathbf{F}-\boldsymbol{\Theta}_{k} \mathbf{C}_{k}\right\|^{2}>\left\|\mathbf{F}-\boldsymbol{\Theta}_{k+1} \mathbf{C}_{k}\right\|^{2} .
$$

Note that if $\mathbf{C}_{k}$ is unitary, then (22) follows directly from (19), since $\left\|\mathbf{F} \mathbf{C}_{k}^{-1}-\boldsymbol{\Theta}_{k}\right\|^{2}=\left\|\mathbf{F}-\boldsymbol{\Theta}_{k} \mathbf{C}_{k}\right\|^{2}$ and $\| \mathbf{F} \mathbf{C}_{k}^{-1}-$ $\boldsymbol{\Theta}_{k+1}\left\|^{2}=\right\| \mathbf{F}-\boldsymbol{\Theta}_{k+1} \mathbf{C}_{k} \|^{2}$. However, when $\mathbf{C}_{k}$ is not unitary, we can still develop further insights by bounding (22). The following proposition is useful in this regard.

Proposition 1: Let $\mathbf{F}, \mathbf{C}_{k}, \boldsymbol{\Theta}_{k}$, and $\boldsymbol{\Theta}_{k+1}$ be defined as above. Let $\mathbf{A}=\mathbf{C}_{k} \mathbf{C}_{k}^{H}$ and

$$
\begin{aligned}
\mathbf{B}=\left(\mathbf{F C}_{k}^{-1}-\boldsymbol{\Theta}_{k}\right)^{H}\left(\mathbf{F C}_{k}^{-1}-\boldsymbol{\Theta}_{k}\right) \\
\quad-\left(\mathbf{F C}_{k}^{-1}-\mathbf{\Theta}_{k+1}\right)^{H}\left(\mathbf{F C}_{k}^{-1}-\mathbf{\Theta}_{k+1}\right) .
\end{aligned}
$$

Then, we have

$$
\begin{aligned}
& \left\|\mathbf{F}-\mathbf{\Theta}_{k} \mathbf{C}_{k}\right\|^{2}-\left\|\mathbf{F}-\mathbf{\Theta}_{k+1} \mathbf{C}_{k}\right\|^{2} \geq \\
& \lambda_{\min }(\mathbf{A}) \operatorname{tr}(\mathbf{B})+\lambda_{\min }(\mathbf{B})\left[\operatorname{tr}(\mathbf{A})-M_{t} \lambda_{\min }(\mathbf{A})\right] .
\end{aligned}
$$

Please refer to Appendix B for the proof of Proposition 1. Note that in (24), we have $\lambda_{\min }(\mathbf{A}) \geq 0$, since $\mathbf{C}_{k} \mathbf{C}_{k}^{H}$ is positive semi-definite, $\operatorname{tr}(\mathbf{B})>0$ from (19), $\left[\operatorname{tr}(\mathbf{A})-M_{t} \lambda_{\min }(\mathbf{A})\right] \geq 0$ and $\lambda_{\min }(\mathbf{B})$ can be either positive or negative. When $\lambda_{\min }(\mathbf{B}) \geq 0$, it is easy to see that (22) holds, since the lower bound in (24) is non-negative. When $\lambda_{\min }(\mathbf{B})<0$, the magnitude of the quantity $(\operatorname{tr}(\mathbf{A})-$ $\left.M_{t} \lambda_{\min }(\mathbf{A})\right)$ decides the sign of the lower bound in (24). Note that $\left(\operatorname{tr}(\mathbf{A})-M_{t} \lambda_{\min }(\mathbf{A})\right)=\sum_{i=1}^{M_{t}}\left(\lambda_{i}(\mathbf{A})-\lambda_{\min }(\mathbf{A})\right)$ measures the spread in the eigenvalues of $\mathbf{A}$. Since the eigenvalues of $\mathbf{A}=\mathbf{C}_{k} \mathbf{C}_{k}^{H}$ are the same as that of $\mathbf{C}_{k}^{H} \mathbf{C}_{k}$, let us take a closer look at this matrix. Since $\mathbf{C}_{k}=\left(\boldsymbol{\Theta}_{k}^{H} \boldsymbol{\Theta}_{k}\right)^{-1} \boldsymbol{\Theta}_{k}^{H} \mathbf{F}$,

\footnotetext{
${ }^{1}$ For more details about the geometric interpretation of subspaces and linear varieties, please refer to Chapter 2 in [26].
}

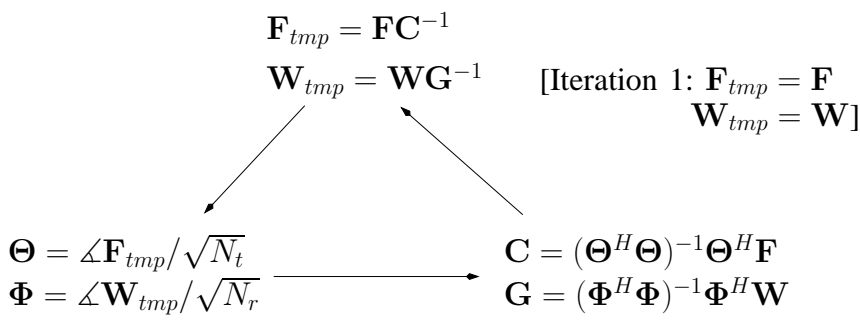

Fig. 1. Pictorial depiction of the IMD iterations.

we have $\mathbf{C}_{k}^{H} \mathbf{C}_{k}=\mathbf{F}^{H} \boldsymbol{\Theta}_{k}\left(\boldsymbol{\Theta}_{k}^{H} \boldsymbol{\Theta}_{k}\right)^{-2} \boldsymbol{\Theta}_{k}^{H} \mathbf{F}$. It can be verified that each of the diagonal entries of $\boldsymbol{\Theta}_{k}^{H} \boldsymbol{\Theta}_{k}$ is 1 and the off-diagonal entries are of the form $\sum_{i=1}^{N_{t}} e^{i \theta_{i}} / N_{t}$. When $N_{t}$ is large, it can be readily seen that the offdiagonal entries tend to zero, hence we have the approximation $\mathbf{C}_{k}^{H} \mathbf{C}_{k} \approx \mathbf{F}^{H} \boldsymbol{\Theta}_{k} \boldsymbol{\Theta}_{k}^{H} \mathbf{F}$. Furthermore, it can be easily verified that the $(i, i)^{\text {th }}$ diagonal entry of $\mathbf{F}^{H} \boldsymbol{\Theta}_{k}$ is $\|\mathbf{F}[:, i]\|_{1} / \sqrt{N_{t}}$ and the $(i, j)^{\text {th }}$ entry is of the form $\sum_{k=1}^{N_{t}}|\mathbf{F}[k, i]|(\measuredangle \mathbf{F}[k, i]$. $\measuredangle \mathbf{F}[k, j]) / \sqrt{N_{t}}$. Again, for large $N_{t}$, the off-diagonal entries become negligible. Thus, based on the Greshgorin circle theorem of [28], we have $\left|\lambda_{i}(\mathbf{A})-\|\mathbf{F}[:, i]\|_{1}^{2} / N_{t}\right|<\epsilon_{i}$, where $\epsilon_{i}$ is the magnitude of the sum of off-diagonal entries in the $i^{\text {th }}$ row of $\mathbf{C}_{k}^{H} \mathbf{C}_{k}$. Since $\{\mathbf{F}[:, i]\}_{i=1}^{M_{t}}$ are orthonormal columns, each of $\|\mathbf{F}[:, i]\|_{1}^{2} / N_{t}$ are nearly identical, when $N_{t}$ is large. Thus, the spread in the eigenvalues $\lambda_{i}(\mathbf{A})$ is nearly zero, hence (22) holds. Furthermore, it may be readily shown that

$$
\left\|\mathbf{F}-\boldsymbol{\Theta}_{k+1} \mathbf{C}_{k}\right\|^{2}>\min _{\mathbf{C}}\left\|\mathbf{F}-\Theta_{k+1} \mathbf{C}\right\|^{2},
$$

and hence we have

$$
\left\|\mathbf{F}-\boldsymbol{\Theta}_{k+1} \mathbf{C}_{k}\right\|^{2}>\left\|\mathbf{F}-\boldsymbol{\Theta}_{k+1} \mathbf{C}_{k+1}\right\|^{2},
$$

where $\mathbf{C}_{k+1}=\arg \min _{\mathbf{C}}\left\|\mathbf{F}-\boldsymbol{\Theta}_{k+1} \mathbf{C}\right\|^{2}$. Thus, from (22) and (25) we have

$$
\begin{gathered}
\left\|\mathbf{F}-\boldsymbol{\Theta}_{k} \mathbf{C}_{k}\right\|^{2}>\left\|\mathbf{F}-\boldsymbol{\Theta}_{k+1} \mathbf{C}_{k+1}\right\|^{2}, \\
\Longrightarrow\left\|\boldsymbol{\Delta}_{k}\right\|^{2}>\left\|\boldsymbol{\Delta}_{k+1}\right\|^{2} .
\end{gathered}
$$

Thus, the residual error in each iteration is non-increasing. Furthermore, we validate (26) with the aid of the simulation results in Section V (see Fig. 7).

\section{B. Comparison with the Existing Schemes}

Most of the existing schemes found in the literature [7]-[14] consider the analog $\mathrm{BF}$ matrices to be beamsteering matrices and iteratively obtain the digital BF matrices by employing Least Squares (LS) weight-optimization. A popular lowcomplexity solution in this set of schemes is the Basis Pursuit based precoding scheme of [8], [9], which uses beamsteering matrices as the analog precoder/combiner and obtains the digital precoder/combiner based on a greedy search method. On the other hand, the authors of [14] propose a simple directional BF approach, where only analog BF matrices are employed, which are obtained based on the dominant channel paths. All these schemes use steering vectors invoked for analog $\mathrm{BF}$, which do not completely exploit the degrees of freedom that the phase shifters offer. Note that each phase shifter can take any value in $[0,2 \pi]$ regardless of the other 

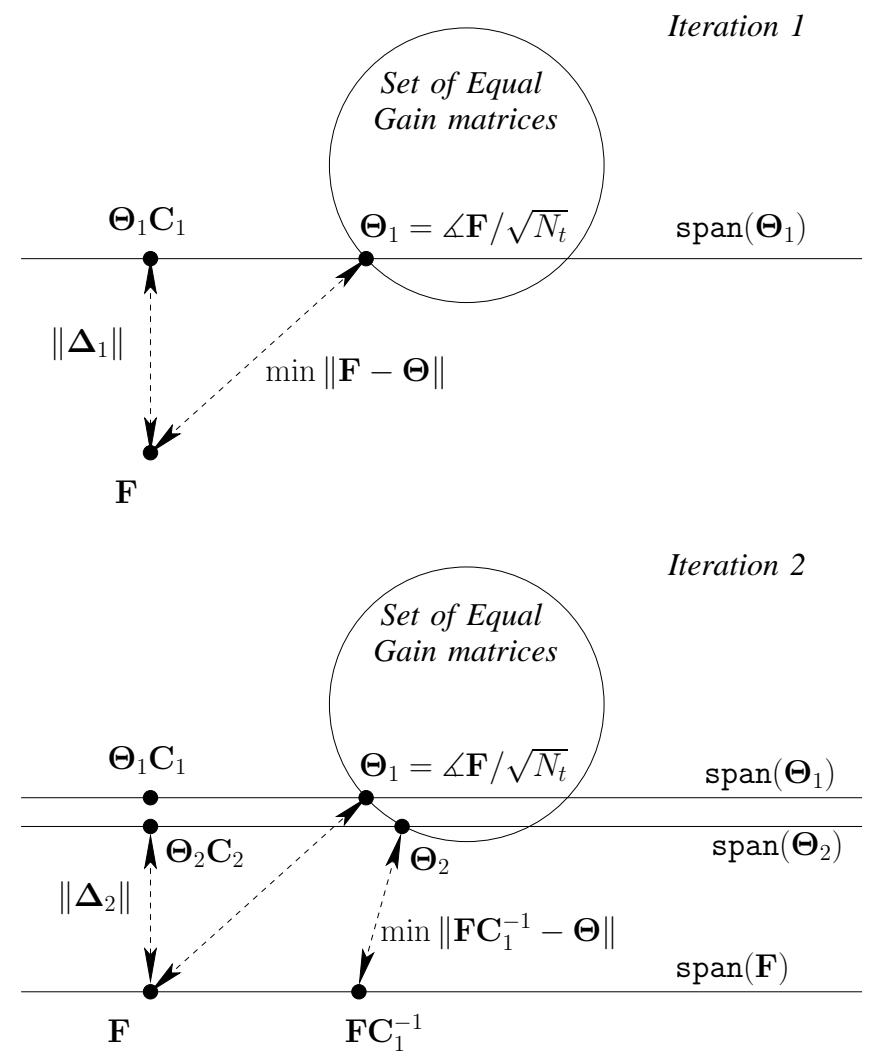

Fig. 2. Geometric interpretation of the IMD iterations

phase shifters, but due to the nature of constant phase offset between the elements in the steering vector, the available DoFs are not completely exploited.

- In the proposed scheme, we have not employed steering vectors, but instead we have used the $\measuredangle(\cdot)$ operator which gives a higher DoF and enables us to more closely approximate the unconstrained solution.

- In contrast to the existing schemes [7]-[14], where the HBF matrix is constructed column-by-column, we consider the entire unconstrained solution (i.e. all columns taken together) and iteratively improve the approximation.

- In contrast to the scheme in [15], the proposed algorithm iteratively improves the hybrid approximation of the digital precoder, while the approximation is a non-iterative single-step procedure in case of the scheme in [15]. Furthermore, the digital precoders of [15] correspond to the finite input alphabet, which is in contrast to the Gaussian input alphabet considered in this paper. Furthermore, the scheme of [15] invokes a computationally demanding gradient ascent method for maximizing the mutual information, while as the proposed solution requires only three simple algebraic steps in each iteration, as shown in Fig. 1.

Owing to the above reasons, the proposed solution attains better performance than the existing solutions, which is confirmed by our simulation results presented in Section V.

Furthermore, the analog phase shifters $\Phi$ and $\Theta$ in Algorithm 1 are assumed to have an infinite resolution, hence, the phase angle of each element in them is assumed to take continuous values in $[0,2 \pi]$. Naturally, practical phase shifters have implementation constraints, hence they are restricted to take discrete phase values. Thus, it is of practical significance to study the performance of Algorithm 1 assuming finiteresolution phase shifters. The performance of Algorithm 1 having phase shifters associated with discrete quantization levels is characterized in Section V.

\section{Proposed Block Diagonalization with UNCONSTRAINED AND CONSTRAINED BF}

In this section, we first show that the knowledge of the AoD of various signal paths between the BS and various UEs is sufficient for block-diagonalizing the mm-wave MUMIMO channel. Then, we propose a novel AoD aided BD method that differs from conventional $\mathrm{BD}$, where the gain of each block-diagonalized channel is improved further by subspace projection, which is equivalent to combining both $\mathrm{BD}$ and $\mathrm{BF}$ to each user. While these methods are primarily proposed for unconstrained systems, we extend these methods to constrained FAS based systems with the aid of our IMD based HBF proposed in Section III.

\section{A. Sufficient CSI for BD}

Considering the unconstrained mm-wave MU-MIMO system given by (5), the composite user channel can be written as

$$
\mathbf{H}_{c o m p}=\left[\mathbf{H}_{1}^{T}, \mathbf{H}_{2}^{T}, \cdots, \mathbf{H}_{K}^{T}\right]^{T} \in \mathbb{C}^{K N_{r} \times N_{t}},
$$

while the precoding matrix can be formulated as

$$
\mathbf{F}=\left[\mathbf{F}_{1}, \mathbf{F}_{2}, \cdots, \mathbf{F}_{K}\right] \in \mathbb{C}^{N_{t} \times K N_{s}},
$$

where $\mathbf{F}_{i} \in \mathbb{C}^{N_{t} \times N_{s}}$ is the precoding matrix associated with the $i^{\text {th }}$ user.

Definition 2: A precoding matrix $\mathbf{F}$ is said to blockdiagonalize the composite user channel $\mathbf{H}_{c o m p}$, if $\mathbf{H}_{i} \mathbf{F}_{j}=$ $\mathbf{O}_{N_{r} \times N_{s}}$ for $1 \leq i \neq j \leq K$.

The following proposition [25] shows that the knowledge of AoD is sufficient for achieving BD.

Proposition 2 ([25] Spencer et. al.): Given a composite user channel $\mathbf{H}_{\text {comp }}$, the knowledge of the AoDs of various users given by $\left\{\mathbf{E}_{t}^{(1)}, \mathbf{E}_{t}^{(2)}, \cdots, \mathbf{E}_{t}^{(K)}\right\}$ is sufficient for obtaining a block-diagonalizing precoder $\mathbf{F}$.

Proof: The composite user channel can be written as

$$
\begin{aligned}
\mathbf{H}_{c o m p} & =\left[\begin{array}{c}
\mathbf{H}_{1} \\
\mathbf{H}_{2} \\
\vdots \\
\mathbf{H}_{K}
\end{array}\right]=\left[\begin{array}{c}
\mathbf{E}_{r}^{(1)} \mathbf{D}^{(1)} \mathbf{E}_{t}^{(1)^{H}} \\
\mathbf{E}_{r}^{(2)} \mathbf{D}^{(2)} \mathbf{E}_{t}^{(2)^{H}} \\
\vdots \\
\mathbf{E}_{r}^{(K)} \mathbf{D}^{(K)} \mathbf{E}_{t}^{(K)^{H}}
\end{array}\right], \\
& =\overline{\mathbf{H}}_{r} \mathbf{E}_{t c o m p},
\end{aligned}
$$


where

$$
\overline{\mathbf{H}}_{r}=\left[\begin{array}{cccc}
\mathbf{E}_{r}^{(1)} \mathbf{D}^{(1)} & \mathbf{O} & \cdots & \mathbf{O} \\
\mathbf{O} & \mathbf{E}_{r}^{(2)} \mathbf{D}^{(2)} & \cdots & \mathbf{O} \\
\vdots & \vdots & \ddots & \vdots \\
\mathbf{O} & \mathbf{O} & \cdots & \mathbf{E}_{r}^{(K)} \mathbf{D}^{(K)}
\end{array}\right]
$$

and $\mathbf{E}_{t c o m p}=\left[\mathbf{E}_{t}^{(1)}, \mathbf{E}_{t}^{(2)}, \ldots, \mathbf{E}_{t}^{(K)}\right]^{H}$. Let $\mathbf{Q}_{j}=$ $\left[\mathbf{E}_{t}^{(1)}, \ldots, \mathbf{E}_{t}^{(j-1)}, \mathbf{E}_{t}^{(j+1)}, \ldots, \mathbf{E}_{t}^{(K)}\right]^{H}=\mathbf{U}_{j} \boldsymbol{\Sigma}_{j} \mathbf{V}_{j}^{H}$ for $1 \leq$ $j \leq K$. Assuming $N_{t} \geq K L$ and $L=N_{s}$ for the sake of simplicity, we opt for

$$
\mathbf{F}_{j}=\mathbf{V}_{j}\left(:,\left[N_{t}-L+1: N_{t}\right]\right) \in \mathbb{C}^{N_{t} \times L},
$$

which is a subset of the null-space basis of $\mathbf{Q}_{j}$. Thus, we have

$$
\mathbf{Q}_{j} \mathbf{F}_{j}=\mathbf{O}_{(K-1) L \times L},
$$

for $1 \leq j \leq K$. Now, considering $\mathbf{H}_{i} \mathbf{F}_{j}$ for $1 \leq i \neq j \leq K$, we have

$$
\begin{aligned}
\mathbf{H}_{i} \mathbf{F}_{j} & =\mathbf{E}_{r}^{(i)} \mathbf{D}^{(i)} \mathbf{E}_{t}^{(i)}{ }^{H} \mathbf{F}_{j}, \\
& =\mathbf{O}_{N_{r} \times L},
\end{aligned}
$$

where the equality in (36) follows from (34). This concludes the proof.

While the precoder of (33) is suitable for blockdiagonalizing the composite user channel, it does not capture the user-signal power effectively. This is so because the null-space basis of $\mathbf{Q}_{j}$ is of dimension $N_{t}-$ $(K-1) L$, from which an $L$ dimensional subspace $\mathbf{V}_{j}\left(:,\left[N_{t}-L+1: N_{t}\right]\right) \in \mathbb{C}^{N_{t} \times L}$ is sought as the precoder. Since $N_{t}$ is very large and $L$ is quite small in mm-wave communication, a naive choice of picking the last $L$ basis vectors as in (33) ([24], [25]) for constructing the precoder would result in very poor performance, since this choice of basis may potentially be orthogonal to the user-signal. This problem can be severe when the number of users $K$ is very small. This issue is overcome by choosing a set of basis vectors in the null-space of $\mathbf{Q}_{j}$ that align with the user-signal's space.

\section{B. Proposed Subspace Projection based AoD aided BD ( $S P$ - $A o D-B D)$}

While it is plausible from Proposition 2 that the knowledge of the AoD of various signal paths between the BS and UEs is sufficient to block-diagonalize the downlink mm-wave MUMIMO channel, the choice of the precoder formulated in (33) [24], [25], does not ensure that the signal energy is maximized in the direction of the intended UE, which would be imperative for achieving reasonable SNRs in mm-wave communication. Let us illustrate this problem with the aid of an example. Consider a three-user scenario with two channel paths between the BS and each of the users, as illustrated in Fig. 3. Let us assume that the BS is capable of transmitting in sixteen orthogonal directions and the channel paths associated with the three users are aligned with the orthogonal directions, as shown in Fig. 3. In conventional BD [25], any set of vectors orthogonal to the directions corresponding to users 1 and 2

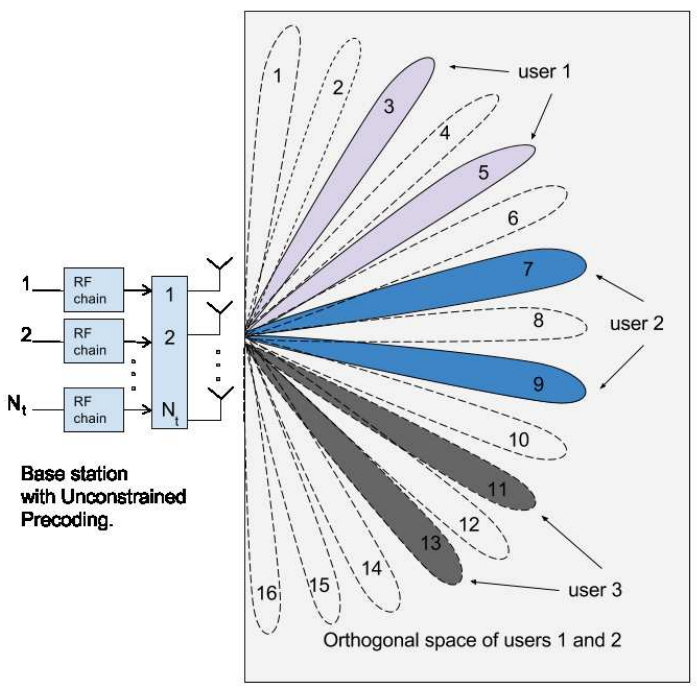

Fig. 3. Pictorial depiction of the signal directions and the interference directions associated with users 1,2 and 3.

(i.e. beams 3, 4, 7 and 9 in Fig. 3) are picked. This could be any beam from 1 to 16 , except those corresponding to users 1 and 2. For example, beams 1 and 6 could be selected, but this would yield nearly zero SNR for user 3 . Thus, among the beams orthogonal to those supporting users 1 and 2, only those beams have to be selected which align with the channel paths of user 3 . This is achieved by projecting the orthogonal beamspace of users 1 and 2 onto the signal space of user 3. The mathematical formulation of the proposed approach is given as follows.

Let $\mathbf{F}_{j}^{\text {(int_null) }}=\mathbf{V}_{j}\left(:,\left[(K-1) L+1: N_{t}\right]\right)$, where $\mathbf{V}_{j}$ is same as that used in (33), $\mathbf{E}_{t}^{(j)}{ }^{H}=\tilde{\mathbf{U}}_{j} \tilde{\mathbf{\Sigma}}_{j} \tilde{\mathbf{V}}_{j}^{H}$, and $\mathbf{F}_{j}^{(\text {sig })}=\tilde{\mathbf{V}}_{j}(:,[1: L])$. Note that $\mathbf{F}_{j}^{\text {(int_null) }}$ corresponds to the orthogonal complement of the interference subspace which spans the entire null-space of $\mathbf{Q}_{j}$ and $\mathbf{F}_{j}^{(\text {sig) }}$ corresponds to the signal space. Let $\mathbf{P}_{j}^{\text {(int_null) }}$ and $\mathbf{P}_{j}^{(\text {sig) }}$ represent the projection matrices associated with the $\mathbf{F}_{j}^{\text {(int_null) }}$ and $\mathbf{F}_{j}^{\text {(sig) }}$, respectively. Considering $\mathbf{P}_{j}^{\text {(eff) }}=\mathbf{P}_{j}^{(\text {sig) }} \mathbf{P}_{j}^{j \text { (int_null) }}=\overline{\mathbf{U}}_{j} \overline{\boldsymbol{\Sigma}}_{j} \overline{\mathbf{V}}_{j}^{H}$, the proposed precoder is given by

$$
\mathbf{F}_{j}=\overline{\mathbf{V}}_{j}(:,[1: L]) \in \mathbb{C}^{N_{t} \times L},
$$

for $1 \leq j \leq K$. The main difference between the precoder proposed in (37) and that of [24], [25], in (33) is that the former depends both on the channels of the intended user as well as of the interfering users, while the latter depends only on the interfering users' channel.

The following proposition shows that the precoder proposed in (37) block-diagonalizes the $\mathbf{H}_{\text {comp }}$.

Proposition 3: Given a composite user channel $\mathbf{H}_{\text {comp }}$, the precoder proposed in (37) satisfies $\mathbf{H}_{i} \mathbf{F}_{j}=\mathbf{O}_{N_{r} \times L}$ for $1 \leq$ $i \neq j \leq K$.

Proof: In order to prove the proposition, it is sufficient to show that the columns of $\mathbf{F}_{j}$ are in the $\operatorname{span}\left(\mathbf{F}_{j}^{\text {(int_null) }}\right)$ for $1 \leq j \leq K$. Since $\mathbf{F}_{j}$ corresponds to the right singular 
vectors of $\mathbf{P}_{j}^{(\text {eff })}$, which are the eigenvectors of $\mathbf{P}_{j}^{(\text {eff })^{H}} \mathbf{P}_{j}^{(\text {eff })}$, we have

$$
\mathbf{P}_{j}^{(\text {eff })^{H}} \mathbf{P}_{j}^{(\text {eff })}=\mathbf{F}_{j}^{(\text {int_null) }} \mathbf{Z}_{j} \mathbf{F}_{j}^{(\text {int_null })^{H}} \in \mathbb{C}^{N_{t} \times N_{t}},
$$

where

$$
\mathbf{Z}_{j}=\mathbf{F}_{j}^{\left(\text {int_null) }{ }^{H}\right.} \mathbf{F}_{j}^{(\text {sig })} \mathbf{F}_{j}^{(\text {sig })^{H}} \mathbf{F}_{j}^{(\text {int_null })} \in \mathbb{C}^{L \times L} .
$$

Upon invoking the eigenvalue decomposition, we have

$$
\mathbf{Z}_{j}=\mathbf{A}_{j} \boldsymbol{\Lambda}_{j} \mathbf{A}_{j}^{H} .
$$

Substituting (40) into (38), we have

$$
\mathbf{P}_{j}^{(\text {eff })^{\mathrm{H}}} \mathbf{P}_{j}^{(\text {eff })}=\mathbf{F}_{j}^{(\text {int_null) }} \mathbf{A}_{j} \mathbf{\Lambda}_{j} \mathbf{A}_{j}^{H} \mathbf{F}_{j}^{(\text {int_null })^{\mathrm{H}}} .
$$

Multiplying both sides of (41) by $\mathbf{F}_{j}^{\text {(int_null) }} \mathbf{A}_{j}$, we have

$$
\mathbf{P}_{j}^{\left(\text {eff }{ }^{H}\right.} \mathbf{P}_{j}^{(\text {eff })} \mathbf{F}_{j}^{(\text {int_null) }} \mathbf{A}_{j}=\mathbf{F}_{j}^{(\text {int_null) }} \mathbf{A}_{j} \mathbf{\Lambda}_{j} .
$$

It is clear from (42) that the eigenvectors of $\mathbf{P}_{j}^{(\text {eff })^{H}} \mathbf{P}_{j}^{(\text {eff) }}$ are in the $\operatorname{span}\left(\mathbf{F}_{j}^{\text {(int_null) }}\right)$. Thus, the columns of $\mathbf{F}_{j}$ are in the $\operatorname{span}\left(\mathbf{F}_{j}^{\text {(int_null) }}\right)$ for $1 \leq j \leq K$. This concludes the proof.

Note that we have assumed $L=N_{s}$ for the ease of presentation, but the proposed solution can be readily extended to the case of $L>N_{s}$. The proposed solution remains the same, except for the choice of the precoder of (37). Instead of choosing the first $L$ basis vectors, we choose first $N_{s}$ basis vectors i.e. $\mathbf{F}_{j}=\overline{\mathbf{V}}_{j}\left(:,\left[1: N_{s}\right]\right) \in \mathbb{C}^{N_{t} \times N_{s}}$ for $1 \leq j \leq K$. The extension of the proposed solution to the constrained FAS based system is identical for both the $L=N_{s}$ and $L>N_{s}$ cases.

Complexity Comparison: Note that we have used an orthonormal basis for the signal space and the interference space for each user, i.e. we have $\mathbf{F}_{j}^{(\text {int_null) }}$ and $\mathbf{F}_{j}^{(\mathrm{sig})}$, for ease of presentation and for comparison with the existing BD schemes. Instead of the above orthonormal basis, we can choose $\mathbf{P}_{j}^{\text {(int_null) }}=\mathbf{I}-\mathbf{Q}_{j}^{H}\left(\mathbf{Q}_{j} \mathbf{Q}_{j}^{H}\right)^{-1} \mathbf{Q}_{j}$ and $\mathbf{P}_{j}^{(\text {sig })}=$ $\mathbf{E}_{t}^{(j)}\left(\mathbf{E}_{t}^{(j)^{H}} \mathbf{E}_{t}^{(j)}\right)^{-1} \mathbf{E}_{t}^{(j)^{H}}$, thereby avoiding the computation of the SVD for obtaining $\mathbf{P}_{j}^{(\text {sig) }}$ and $\mathbf{P}_{j}^{\text {(int_null) }}$. Thus, the solution proposed for an unconstrained system requires only one SVD computation per user, which is the same as that required by the existing BD scheme of [24], [25]. Considering the fact that the BD scheme is employed at the BS, which in general is not limited by the power or by the computational capability, we believe that the additional computation of the projection matrices in the proposed approach does not impose a serious burden.

\section{Subspace Projection based AoD aided BD combined with $H B F(S P-B D-H B F)$}

So far we considered an unconstrained system, where $N_{t}$ $\mathrm{RF}$ chains are assumed to be present. Let us now consider a constrained FAS based system as described in Section II-C, which has fewer RF chains than the number of antennas.

Given the SP-AoD-BD precoder $\overline{\mathbf{V}}_{j}(:,[1: L])$ (37) for the $j^{\text {th }}$ user, we obtain $\boldsymbol{\Theta}_{j}$ and $\mathbf{C}_{j}$ by using IMD based HBF (see Algorithm 1) that approximate $\overline{\mathbf{V}}_{j}(:,[1: L])$ i.e. $\overline{\mathbf{V}}_{j}($ : , $[1: L]) \approx \boldsymbol{\Theta}_{j} \mathbf{C}_{j}$. Although, we have $\mathbf{H}_{i} \overline{\mathbf{V}}_{j}(:,[1: L])=$ $\mathbf{O}_{N_{r} \times L}, \mathbf{H}_{i} \boldsymbol{\Theta}_{j} \mathbf{C}_{j} \neq \mathbf{O}_{N_{r} \times L}$ due to the residual error in the approximation defined in Section III-A. We resort to BD in the digital domain to handle the residual interference, whose details are given as follows.

Let the baseband composite user channel be represented by

$$
\mathbf{K}_{c o m p}=\left[\begin{array}{c}
\mathbf{K}_{1} \\
\mathbf{K}_{2} \\
\vdots \\
\mathbf{K}_{K}
\end{array}\right] \in \mathbb{C}^{K L \times K L}
$$

where $\mathbf{K}_{i}=\left[\mathbf{E}_{t}^{(i)}{ }^{H} \boldsymbol{\Theta}_{1} \mathbf{C}_{1}, \mathbf{E}_{t}^{(i)}{ }^{H} \boldsymbol{\Theta}_{2} \mathbf{C}_{2}, \ldots, \mathbf{E}_{t}^{(i)^{H}} \boldsymbol{\Theta}_{K} \mathbf{C}_{K}\right]$ for $1 \leq i \leq K$. Let furthermore $\mathbf{R}_{j}=$ $\left[\mathbf{K}_{1}^{T}, \ldots, \mathbf{K}_{j-1}^{T}, \mathbf{K}_{j+1}^{T}, \ldots, \mathbf{K}_{K}^{T}\right]^{T}=\check{\mathbf{U}}_{j} \check{\Sigma}_{j} \check{\mathbf{V}}_{j}^{H}$ and $\mathbf{J}_{j}=\check{\mathbf{V}}_{j}(:,[(K-1) L+1: K L]) \in \mathbb{C}^{K L}$ for $1 \leq j \leq K$. It may then be observed that $\mathbf{K}_{i} \mathbf{J}_{j}=\mathbf{O}_{L \times L}$ for $1 \leq i \neq j \leq K$. Thus, the effective pre-processing invoked for achieving BD at the BS is given by

$$
\left[\boldsymbol{\Theta}_{1}, \boldsymbol{\Theta}_{2}, \ldots, \boldsymbol{\Theta}_{K}\right]\left[\begin{array}{cccc}
\mathbf{C}_{1} & \mathbf{O} & \cdots & \mathbf{O} \\
\mathbf{O} & \mathbf{C}_{2} & \cdots & \mathbf{O} \\
\vdots & \vdots & \ddots & \vdots \\
\mathbf{O} & \mathbf{O} & \cdots & \mathbf{C}_{K}
\end{array}\right]\left[\mathbf{J}_{1}, \mathbf{J}_{2}, \ldots, \mathbf{J}_{K}\right] .
$$

Given this pre-processing, the effective channel as seen by the $j^{\text {th }}$ user becomes

$$
\mathbf{H}_{j}^{(\mathrm{eff})}=\mathbf{E}_{r}^{(j)} \mathbf{D}^{(j)} \mathbf{K}_{j} \mathbf{J}_{j} \in \mathbb{C}^{N_{r} \times L} .
$$

The optimal combining and precoding matrix conditioned for the $j^{\text {th }}$ user corresponds to the left and right singular vectors associated with dominant singular values of $\mathbf{H}_{j}^{\text {(eff) }}$, respectively. If $\mathbf{H}_{j}^{\text {(eff) }}=\breve{\mathbf{U}}_{j} \breve{\mathbf{\Sigma}}_{j} \breve{\mathbf{V}}_{j}^{H}$, then the unconstrained combining matrix is taken to be $\mathbf{W}_{j}=\breve{\mathbf{U}}_{j}(:,[1: L])$. The analog and digital combining matrices derived for HBF are obtained using Algorithm 1, which approximates the unconstrained solution as $\breve{\mathbf{U}}_{j}(:,[1: L]) \approx \boldsymbol{\Phi}_{j} \mathbf{G}_{j}$. Each user acquires the CSI with the aid of downlink channel training, where the BS transmits training signals over each block-diagonalized user channel. Upon acquiring the channel knowledge, each UE computes $\overrightarrow{\mathbf{V}}_{j}$ and conveys it back to the BS. The BS uses $\breve{\mathbf{V}}_{j}$ in order to diagonalize the $j^{\text {th }}$ user channel. Thus, the effective pre-processing invoked for $\mathrm{BD}$ and user channel diagonalization is

$$
\left[\boldsymbol{\Theta}_{1} \mathbf{C}_{1}, \boldsymbol{\Theta}_{2} \mathbf{C}_{2}, \ldots, \boldsymbol{\Theta}_{K} \mathbf{C}_{K}\right]\left[\mathbf{J}_{1} \breve{\mathbf{V}}_{1}, \mathbf{J}_{2} \breve{\mathbf{V}}_{2}, \ldots, \mathbf{J}_{K} \breve{\mathbf{V}}_{K}\right]
$$

The steps involved in establishing a reliable downlink are summarized as follows:

1) The BS acquires the AoD knowledge of the channel paths associated with each user. This can be achieved by uplink channel sounding as detailed in [18], [19].

2) Upon obtaining the AoD knowledge, the BS obtains the effective pre-processing matrix given by (44) that allows to establish an interference free channel to each of the users. 
3) With the aid of downlink channel training over interference free channels, each user acquires the knowledge of $\mathbf{H}_{j}^{(e f f)}$ and obtains the combining matrix $\breve{\mathbf{U}}_{j}$ and then signals $\breve{\mathbf{V}}_{j}$ back to the BS.

4) Upon obtaining the knowledge of $\breve{\mathbf{V}}_{j}$, the BS uses the pre-processing matrix given by (46) for downlink data transmission.

In this paper, we assume that all the parameters estimated in the above steps are perfect, and study the impact of imperfect estimation on the attainable system performance in our future work. Assuming a Gaussian alphabet and equalpower allocation across all the user streams by the BS, the total sum of the spectral efficiency [31] is given by

$$
R=\sum_{i=1}^{K} \log _{2}\left(\left|\mathbf{I}_{L}+\frac{P}{K L}\left(\mathbf{W}_{i}^{H} \mathbf{W}_{i}\right)^{-1} \mathbf{W}_{i}^{H} \mathbf{H}_{i}^{(\text {eff })} \mathbf{H}_{i}^{\left(\text {eff }{ }^{H}\right.} \mathbf{W}_{i}\right|\right)
$$

which is measured in terms of bits per channel use (bpcu). By contrast, in the unconstrained system, we have $\mathbf{W}_{i}=\mathbf{U}_{i}$ and hence

$$
R=\sum_{i=1}^{K} \log _{2}\left(\left|\mathbf{I}_{L}+\frac{P}{K L} \breve{\mathbf{U}}_{i}^{H} \mathbf{H}_{i}^{(\text {eff })} \mathbf{H}_{i}^{\left(\text {eff }{ }^{H}\right.} \breve{\mathbf{U}}_{i}\right|\right) .
$$

In the constrained FAS based system, we have $\mathbf{W}_{i}=\boldsymbol{\Phi}_{i} \mathbf{G}_{i}$ and hence

$$
\begin{aligned}
R=\sum_{i=1}^{K} \log _{2}\left(\mid \mathbf{I}_{L}+\frac{P}{K L}\left(\mathbf{G}_{i}^{H} \boldsymbol{\Phi}_{i}^{H} \boldsymbol{\Phi}_{i} \mathbf{G}_{i}\right)^{-1}\right. \\
\left.\mathbf{G}_{i}^{H} \boldsymbol{\Phi}_{i}^{H} \mathbf{H}_{i}^{(\mathrm{eff})} \mathbf{H}_{i}^{(\mathrm{eff})^{H}} \boldsymbol{\Phi}_{i} \mathbf{G}_{i} \mid\right) .
\end{aligned}
$$

Comparison with the Existing Schemes: We contrast the proposed scheme to those of [19] and [20]. Both these schemes independently choose the analog BF matrices and the digital BD precoders in two different steps in an independent fashion. We refer to the scheme proposed in [19] as the two-stage multi-user hybrid BF (TS-MU-HBF) and that proposed in [20] as the equal gain transmission based hybrid BF (EGTHBF). In the first stage of TS-MU-HBF, $\left\{\boldsymbol{\Theta}_{i}, \boldsymbol{\Phi}_{i}\right\}$ are obtained jointly from a finite set of quantized beamsteering vectors that maximize the baseband channel gain of $\left\|\boldsymbol{\Phi}_{i}^{H} \mathbf{H}_{i} \boldsymbol{\Theta}_{i}\right\|$ for each user $i=1,2, \ldots, K$. In the second stage of TS-MU-HBF, the conventional $\mathrm{BD} / \mathrm{ZF}$ precoding [24] that diagonalizes the composite baseband user channel is employed. The preprocessing stages at the BS are identical to that in (44), except that the $\mathbf{C}_{i}$ represents identity matrices. Note that the analog phaseshifters at the BS $\left[\Theta_{1}, \Theta_{2}, \ldots, \Theta_{K}\right]$ are used in a fashion dedicated to each user in both the proposed as well as the TS-MUHBF schemes. In EGT-HBF, the analog combiners $\boldsymbol{\Phi}_{i}$ of the receivers are chosen from a DFT codebook that maximize the received energy $\left\|\Phi_{i}^{H} \mathbf{H}_{i}\right\|$, while the analog precoders are obtained based on the equal gain transmission method [22]. Explicitly, $\boldsymbol{\Theta}_{i}$ is chosen to be $\measuredangle\left(\mathbf{H}_{i}^{H} \boldsymbol{\Phi}_{i}\right) / \sqrt{N_{t}}$. Upon obtaining the analog precoders and combiners, the baseband channel is diagonalized by employing the conventional BD scheme [24]. Note that even in the case of EGT-HBF, the analog precoders $\left[\boldsymbol{\Theta}_{1}, \boldsymbol{\Theta}_{2}, \ldots, \boldsymbol{\Theta}_{K}\right]$ of the BS are used in a fashion dedicated to each user and the preprocessing stages at the BS are identical to that in (44), except that the $\mathbf{C}_{i}$ represents identity matrices.

\section{Simulation Results and Discussions}

In this section, we study the achievable spectral efficiency of the proposed IMD based $\mathrm{HBF}$ as well as that of the proposed AoD-BD and SP-AoD-BD both in unconstrained and constrained BF scenarios.

Simulation scenario: In all our simulations, we assume the geometric channel model of Section II-A. Both the BS and all the UEs are assumed to have their antenna elements arranged in ULA with an inter-element spacing of $d=\lambda / 2$. The spectral efficiency curves are obtained by averaging over one thousand channel realizations at each SNR point.

\section{A. IMD based $\mathrm{HBF}$}

Consider a single-user mm-wave MIMO system having $N_{t}=N_{r} \in\{8,16,32,64\}, M_{t}=M_{r}=N_{s}=2$. Let the number of channel clusters be $L=6$. Fig. 4 portrays the achievable rates of both the unconstrained and of the existing schemes as well as of the proposed IMD-HBF as a function of the SNR. It is clear from Fig. 4 that the proposed IMD-HBF achieves nearly the same rate as that of the unconstrained system even when the number of antennas is not large, while the existing solutions suffer from a performance loss. Furthermore, an SNR gain of about 6 $\mathrm{dB}$ is observed, when the number of antennas is doubled, which corresponds to the array gain. Let us now quantify the achievable gain in the proposed solution w.r.t. the existing schemes at a particular SNR. Fig. 5 compares the performance of the aforementioned schemes, when operating at an SNR of $-5 \mathrm{~dB}$ for $N_{t}=N_{r} \in\{8,16,32,64\}$. It is evident from Fig. 5 that the proposed solution achieves significantly better performance compared to the existing schemes and requires only about ten iterations to converge. Specifically, in a system having $N_{t}=N_{r}=8$, the proposed scheme achieves a gain of about 0.7 bpcu w.r.t. the Basis Pursuit based scheme [8], [9], and a gain of about 0.85 bpcu w.r.t. the beamsteering based on dominant directions [14].

Let us now study the performance of the proposed IMDHBF scheme considering a fixed $N_{t}=N_{r}=64$ and various values of $M_{t}=M_{r} \in\{2,3,4,5,6\}$, when operating in a channel having $L=6$ clusters. Fig. 6 portrays the achievable rates of both unconstrained and of the IMD-HBF system, when operating with the aid of the aforementioned system parameters. It is evident from Fig. 6 that the proposed IMD$\mathrm{HBF}$ does not suffer from any significant performance loss and achieves nearly the same rate as that attained by the unconstrained system. Fig. 7 portrays the reduction in the approximation error [(9), (10)] as a function of the number of IMD iterations. It is evident from Fig. 7 that as the value of $M_{t}=M r$ is increased, the approximation error reduces significantly for any number of IMD iterations. This is as expected, since a larger value of $M_{t}=M_{r}$ results in a higher dimensional subspace for approximating the singular vectors better, which lie in the space spanned by the $L$ steering vectors. When $M_{t}=M_{r}=L$, the approximation error is 


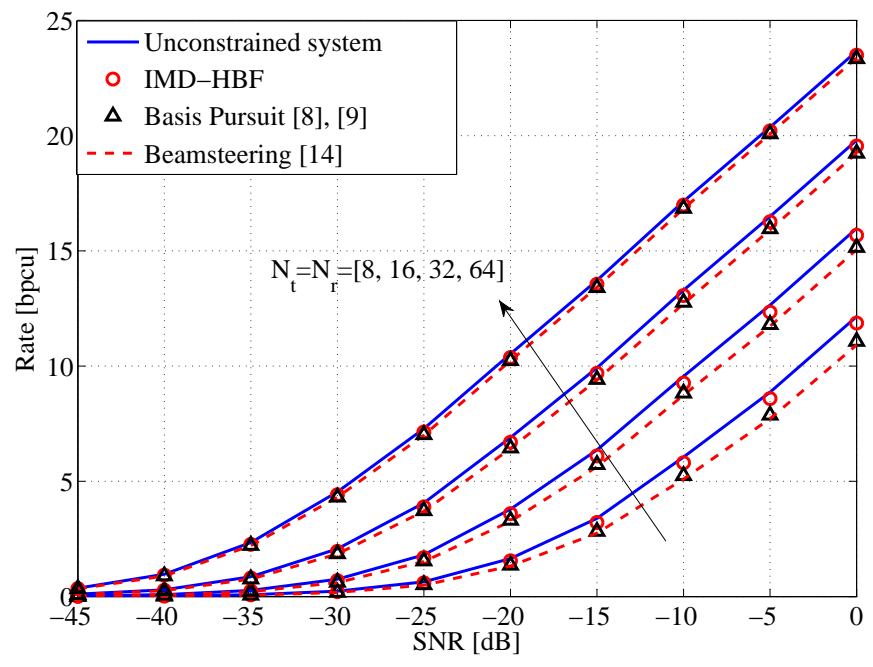

Fig. 4. Comparison of the achievable rates in the unconstrained system having $N_{t}=N_{r}=M_{t}=M_{r}$ with that of the IMD-HBF and the existing schemes having $M_{t}=M_{r}=N_{s}=2$ for various values of $N_{t}$ and $N_{r}$. The number of channel clusters is $L=6$ and the number of iterations in the IMD-HBF is taken to be 40 .

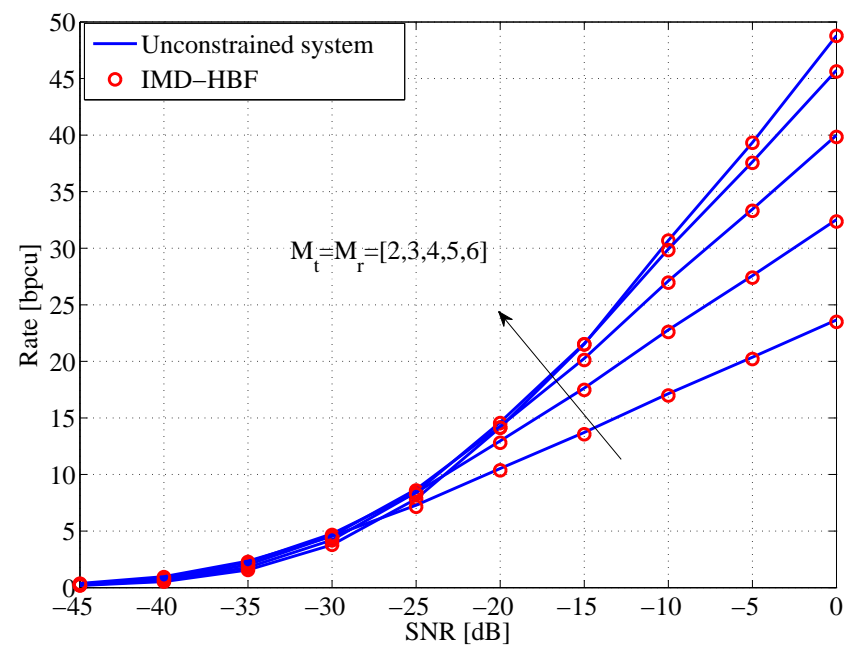

Fig. 6. Comparison of the achievable rates in the unconstrained system having $N_{t}=N_{r}=M_{t}=M_{r}=64$ with that of the IMD-HBF system having $N_{t}=N_{r}=64$ for various values of $M_{t}=M_{r} \in\{2,3,4,5,6\}$. The channel is assumed to have $L=6$ clusters and the number of IMD iterations in the IMD-HBF is taken to be 40 .

less than $96 \%$ and the achievable rate coincides with that of the unconstrained system. Furthermore, it is evident from Fig. 7 that a maximum of 10 IMD iterations are sufficient for the algorithm to converge, which renders it a low-complexity solution.

So far we have assumed that the analog phase shifters employed in HBF systems have an infinite resolution. Naturally, this is not the case in practical systems, since the variable phase shifters can only assume discrete phase values. Fig. 8 compares the achievable rate of an HBF system having $N_{t}=N_{r}=64, M_{t}=M_{r}=N_{s}=2$ and employing phase shifters having discrete quantization levels to that of

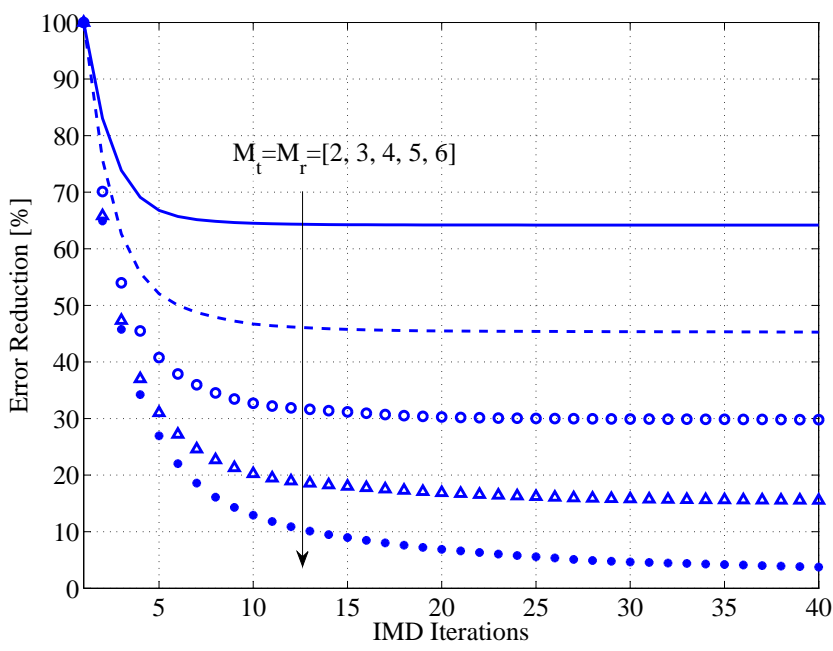

Fig. 7. Variation of the residual error [(9), (10)] as a function of the number of iterations in the IMD-HBF system having $M_{t}=M_{r}=N_{s}=2$ and $N_{t}=N_{r}=64$, when operating in a channel having $L=6$ clusters.
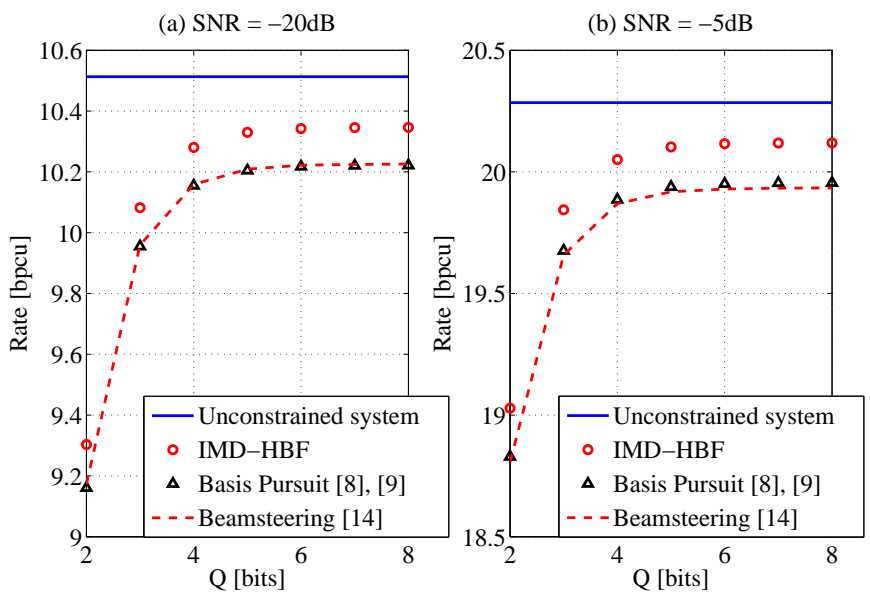

Fig. 8. Variation of the achievable rates in the IMD-HBF as well as the existing schemes as a function of the number of quantization bits of the analog phase shifters associated with precoding and combining matrices. The system is assumed to have $N_{t}=N_{r}=64, M_{t}=M_{r}=N_{s}=2$, and operating in channel having $L=6$ clusters.

the unconstrained system at two specific SNR values. It is evident from Fig. 8(a) and Fig. 8(b) that the proposed IMDHBF associated with five quantization bits achieve a similar performance to that achieved by the system operating with the aid of infinite-resolution phaseshifters.

\section{B. BD with Unconstrained and Constrained BF}

Consider a multi-user mm-wave system, where the BS has $N_{t}=64, M_{t}=K N_{s}$ and each UE has $N_{r}=16$ and $M_{r}=N_{s}=L=2$. First, we compare the performance of the conventional BD to that of the proposed SP-AoD-BD in the above mentioned set-up. Fig. 9 compares the achievable sum rate of the proposed SP-AoD-BD to that of the conventional $\mathrm{BD}$ [25] for various values of $K$. It is evident that the SP-AoD$\mathrm{BD}$ achieves significantly higher rates than the conventional 

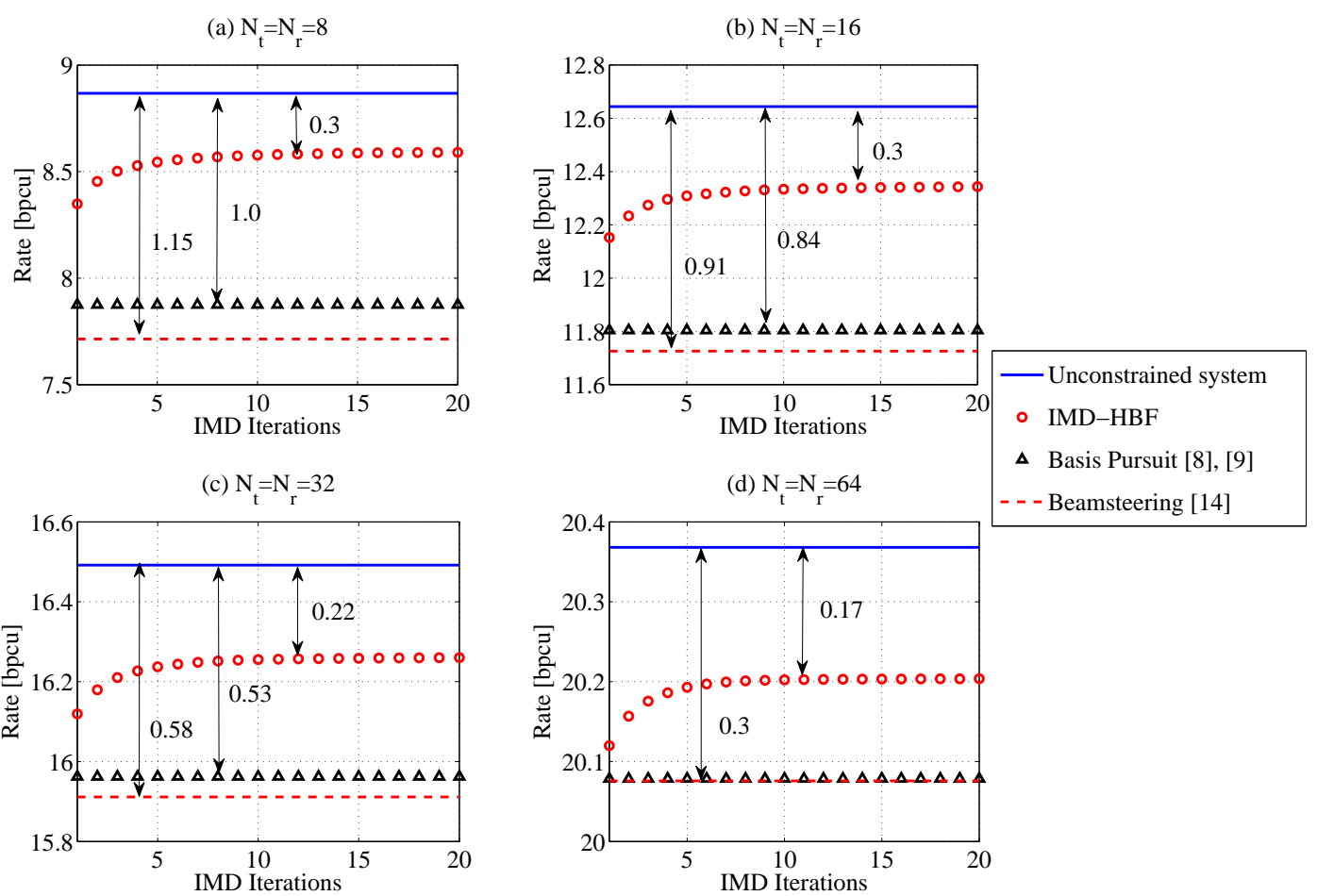

Fig. 5. Comparison of the achievable rates in the unconstrained system having $N_{t}=N_{r}=M_{t}=M_{r}$ with that of the IMD-HBF and the existing schemes having $M_{t}=M_{r}=N_{s}=2$ for various values of $N_{t}$ and $N_{r}$ at $\mathrm{SNR}=-5 \mathrm{~dB}$. The number of channel clusters is $L=6$.

BD. This is not surprising, since the conventional BD does not ensure that the BF vectors are aligned with the AoDs of the channel paths, which is essential for achieving reasonable values of SNR in mm-wave communication.

In the rest of our simulation results,

- we consider SP-AoD-BD using unconstrained BF as well as the EGT-HBF scheme [20] as the benchmark schemes for comparison,

- each of the UEs is assumed to have $N_{r}=16, M_{r}=$ $N_{s}=2$ and $L=\{2,3,4\}$,

- the BS is assumed to have $M_{t}=N_{s} K=2 K$.

Fig. 10 compares the achievable sum rate of the proposed SP-BD-HBF both to that of its unconstrained counterpart as well as to the EGT-HBF [20] and to the TU-MU-HBF [19] schemes as a function of the number of users $K$ at SNR values of $0 \mathrm{~dB},-10 \mathrm{~dB}$, and $-20 \mathrm{~dB}$ for various values of $L$. It is seen from Fig. 10 that the proposed scheme attains a significantly better performance than the existing EGT-HBF scheme, when we have $L=N_{s}$. By contrast, for $L>N_{s}$ the proposed scheme suffers from a performance degradation w.r.t. the EGT-HBF for larger values of $K$, which is mainly due to the suppression of the signal in the user signal space. Specifically, when $K=10$ and SNR $=0 \mathrm{~dB}$ the proposed scheme achieves

- a gain of 18 bpcu in case of $L=2$,

- a gain of 4 bpcu in case of $L=3$, and

- a loss of 5 bpcu in case of $L=4$,

with respect to the EGT-HBF scheme of [20] and

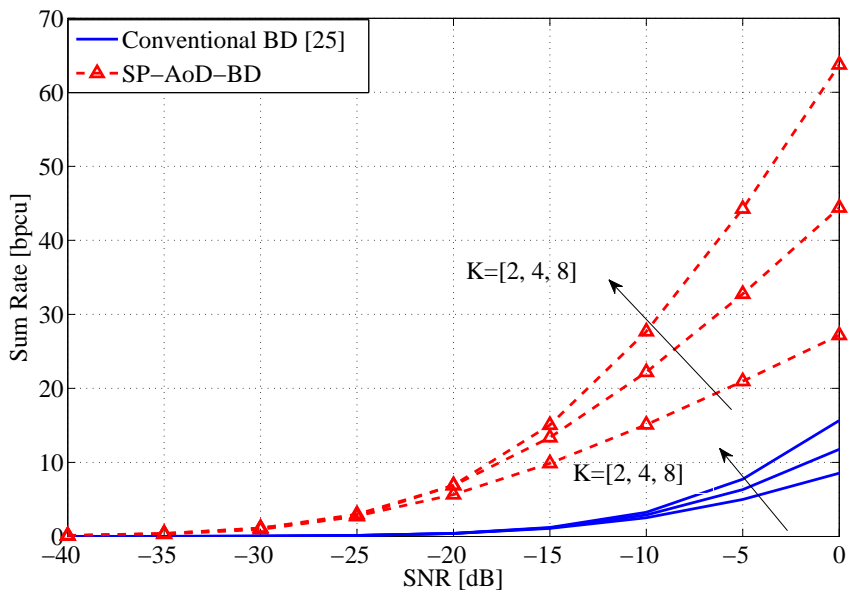

Fig. 9. Comparison of the achievable sum rate in the conventional $\mathrm{BD}$ and the proposed SP-AoD-BD with unconstrained BF in a system having $N_{t}=64, N_{r}=16, M_{t}=K N_{s}, N_{s}=L=M_{r}=2$.

- a gain of 36 bpcu in case of $L=2$,

- a gain of 36 bpcu in case of $L=3$, and

- a loss of 31 bpcu in case of $L=4$,

with respect to the TS-MU-HBF scheme $[19]^{2}$. It is also evident from Fig. 10 that the proposed scheme does not suffer

\footnotetext{
${ }^{2}$ We have used a DFT codebook for analog BF matrices and jointly obtained the precoding/combining matrices based on maximizing the effective baseband channel power, as in Algorithm 1 of [19].
} 
(a) $\mathrm{L}=2$

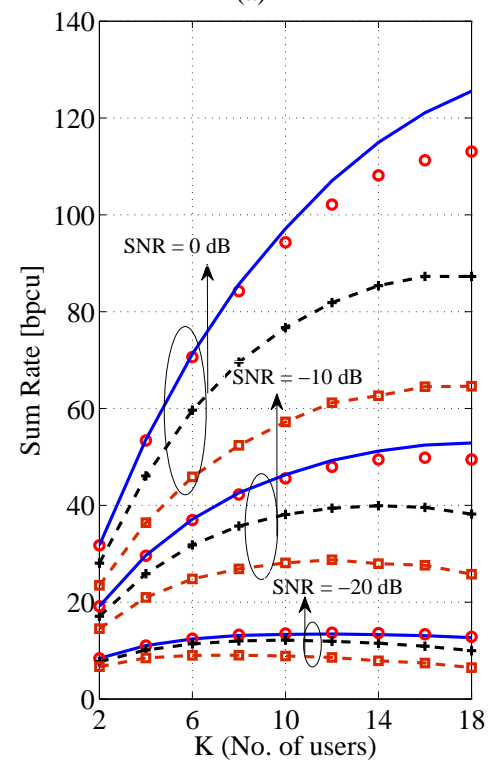

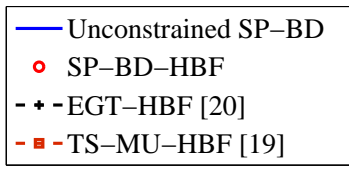

(b) $\mathrm{L}=3$

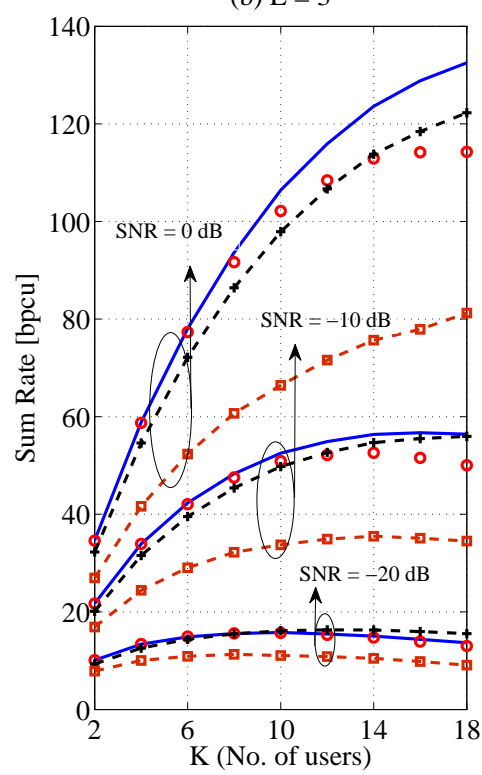

(c) $\mathrm{L}=4$

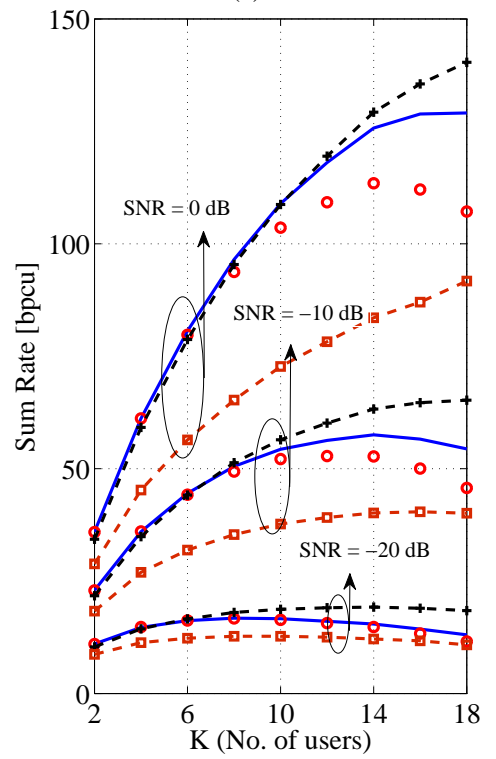

Fig. 10. Comparison of the achievable sum rate as a function of the number of users $K$ at SNR values of $0 \mathrm{~dB},-10 \mathrm{~dB}$, and $-20 \mathrm{~dB}$, in a multi-user mm-wave system, where the BS has $N_{t}=128$ and employs the SP-AoD-BD with unconstrained BF and IMD-HBF as well as the EGT-HBF [20] and TS-MU-HBF [19] schemes. Plots (a)-(c) correspond to the scenarios where the number of channel clusters $L$ ranges from 2 to 4 .

from any performance loss for lower values of $K$, even when $L$ is large. Note that the performance loss encountered in case of larger values of $K$ w.r.t. the EGT-HBF scheme can be significantly reduced by increasing the number of transmit antennas $N_{t}$.

\section{CONCLUSIONS}

We have proposed a low-complexity iterative matrix decomposition based hybrid beamforming algorithm for single-user mm-wave communication, which is capable of achieving the same spectral efficiency as that of the unconstrained SVD based solution. Furthermore, we have shown that the AoD knowledge of channel paths associated with various users is sufficient in order to block-diagonalize the downlink mm-wave channel. We have proposed a subspace projection based AoD aided $\mathrm{BD}$ that gives a significantly better performance than the conventional $\mathrm{BD}$. We have studied the sum rate performance of the proposed SP-AoD-BD in both an unconstrained BF scenario as well as in constrained $\mathrm{BF}$ scenarios, where the proposed IMD-HBF is employed. Furthermore, it is observed that nearly the same performance as that of full-CSI based schemes is attainable by the proposed scheme for moderate number of users. For a fixed number of users, we have observed that the sum rate performance of the constrained BF solution approaches that of the unconstrained solution, when the number of BS antennas is increased. Thus, future multiuser mm-wave communication systems can be designed with the aid of partial CSI whilst achieving reasonably good performance.

\section{APPENDIX A}

Proof: For the ease of presentation, let us denote $\mathbf{F} \mathbf{C}_{k}^{-1}$ by $\tilde{\mathbf{F}}_{k}$. Then, from (17) we have

$$
\begin{aligned}
& \boldsymbol{\Theta}_{k+1}=\underset{|\boldsymbol{\Theta}(i, j)|=1 / \sqrt{N_{t}}}{\arg \min }\left\|\mathbf{F} \mathbf{C}_{k}^{-1}-\boldsymbol{\Theta}\right\|^{2} \\
&=\underset{|\boldsymbol{\Theta}(i, j)|=1 / \sqrt{N_{t}}}{\arg \min }\left\|\tilde{\mathbf{F}}_{k}-\boldsymbol{\Theta}\right\|^{2} \\
&=\underset{|\boldsymbol{\Theta}(i, j)|=1 / \sqrt{N_{t}}}{\arg \min } \sum_{i=1}^{M_{t}} \sum_{j=1}^{N_{t}}\left|\tilde{\mathbf{F}}_{k}(i, j)-\boldsymbol{\Theta}(i, j)\right|^{2} \\
&=\arg \sum_{i=1}^{M_{t}} \sum_{j=1}^{N_{t}} \mid \boldsymbol{\Theta} \\
& \min _{(i, j) \mid=1 / \sqrt{N_{t}}}\left|\tilde{\mathbf{F}}_{k}(i, j)-\boldsymbol{\Theta}(i, j)\right|^{2}
\end{aligned}
$$

where the interchange of summation and minimization follows from the assumption of independent phase shifters in the FAS based system. Thus, it is sufficient to show that

$$
\measuredangle \tilde{\mathbf{F}}_{k}(i, j) / \sqrt{N_{t}}=\underset{|\Theta(i, j)|=1 / \sqrt{N_{t}}}{\arg \min }\left|\tilde{\mathbf{F}}_{k}(i, j)-\boldsymbol{\Theta}(i, j)\right|^{2} .
$$


Letting $\tilde{\mathbf{F}}_{k}(i, j)=\alpha e^{j \beta}$ and $\boldsymbol{\Theta}(i, j)=\gamma e^{j \theta}$, where $\gamma=$ $1 / \sqrt{N_{t}}$, we have

$$
\begin{aligned}
f_{i, j} & =\left|\tilde{\mathbf{F}}_{k}(i, j)-\boldsymbol{\Theta}(i, j)\right|^{2}, \\
& =(\alpha \cos \beta-\gamma \cos \theta)^{2}+(\alpha \sin \beta-\gamma \sin \theta)^{2} .
\end{aligned}
$$

Furthermore, differentiating $f_{i, j}$ w.r.t. $\theta$ and equating to zero gives

$$
\frac{\partial f_{i, j}}{\partial \theta}=2 \alpha \gamma \cos \beta \sin \theta_{o p t}-2 \alpha \gamma \sin \beta \cos \theta_{o p t}=0,
$$

which yields $\theta_{\text {opt }}=\beta$. Furthermore, it can be verified that the optimal solution $\theta_{\text {opt }}$ corresponds to the minimum of $f_{i, j}$, as follows. Considering the second-order derivative of $f_{i, j}$, we have

$$
\begin{aligned}
\frac{\partial^{2} f_{i, j}}{\partial \theta^{2}} & =2 \alpha \gamma \cos \beta \cos \theta+2 \alpha \gamma \sin \beta \sin \theta, \\
\left.\frac{\partial^{2} f_{i, j}}{\partial \theta^{2}}\right|_{\theta=\theta_{o p t}} & =2 \alpha \gamma \cos ^{2} \beta+2 \alpha \gamma \sin ^{2} \beta, \\
& =2 \gamma \alpha>0,
\end{aligned}
$$

which implies that $\theta_{\text {opt }}$ is a minimum of $f_{i, j}$. Similar arguments also hold for the case of (18). This concludes the proof.

\section{APPEndix B}

Proof: We have

$$
\begin{aligned}
& \left\|\mathbf{F}-\boldsymbol{\Theta}_{k} \mathbf{C}_{k}\right\|^{2}-\left\|\mathbf{F}-\boldsymbol{\Theta}_{k+1} \mathbf{C}_{k}\right\|^{2} \\
& =\left\|\left(\mathbf{F C}_{k}^{-1}-\boldsymbol{\Theta}_{k}\right) \mathbf{C}_{k}\right\|^{2}-\left\|\left(\mathbf{F C}_{k}^{-1}-\boldsymbol{\Theta}_{k+1}\right) \mathbf{C}_{k}\right\|^{2}, \\
& =\operatorname{tr}\left\{\mathbf{C}_{k}^{H}\left(\mathbf{F C}_{k}^{-1}-\boldsymbol{\Theta}_{k}\right)^{H}\left(\mathbf{F C}_{k}^{-1}-\boldsymbol{\Theta}_{k}\right) \mathbf{C}_{k}\right\} \\
& -\operatorname{tr}\left\{\mathbf{C}_{k}^{H}\left(\mathbf{F C}_{k}^{-1}-\boldsymbol{\Theta}_{k+1}\right)^{H}\left(\mathbf{F C}_{k}^{-1}-\mathbf{\Theta}_{k+1}\right) \mathbf{C}_{k}\right\} \text {, } \\
& =\operatorname{tr}\left[\left\{\left(\mathbf{F C}_{k}^{-1}-\mathbf{\Theta}_{k}\right)^{H}\left(\mathbf{F C}_{k}^{-1}-\mathbf{\Theta}_{k}\right)\right.\right. \\
& \left.\left.-\left(\mathbf{F C}_{k}^{-1}-\boldsymbol{\Theta}_{k+1}\right)^{H}\left(\mathbf{F C}_{k}^{-1}-\mathbf{\Theta}_{k+1}\right)\right\} \mathbf{C}_{k} \mathbf{C}_{k}^{H}\right], \\
& =\operatorname{tr}\left[\mathbf { C } _ { k } \mathbf { C } _ { k } ^ { H } \left\{\left(\mathbf{F C}_{k}^{-1}-\mathbf{\Theta}_{k}\right)^{H}\left(\mathbf{F C}_{k}^{-1}-\mathbf{\Theta}_{k}\right)\right.\right. \\
& \left.\left.-\left(\mathbf{F C}_{k}^{-1}-\boldsymbol{\Theta}_{k+1}\right)^{H}\left(\mathbf{F C}_{k}^{-1}-\boldsymbol{\Theta}_{k+1}\right)\right\}\right], \\
& =\operatorname{tr}(\mathbf{A B}) \\
& \geq \lambda_{\min }(\mathbf{A}) \operatorname{tr}(\mathbf{B})+\lambda_{\min }(\mathbf{B})\left[\operatorname{tr}(\mathbf{A})-M_{t} \lambda_{\min }(\mathbf{A})\right] .
\end{aligned}
$$

The inequality in (66) follows from [27]. This concludes the proof.

\section{REFERENCES}

[1] Z. Pi and F. Khan, "An introduction to millimeter-wave mobile broadband systems," IEEE Communications Magazine, vol. 49, no. 6, pp. 101-107, 2011.

[2] T. Rappaport, S. Sun, R. Mayzus, H. Zhao, Y. Azar, K. Wang, G. N. Wong, J. K. Schulz, M. Samimi and F. Gutierrez "Millimeter wave mobile communications for $5 \mathrm{G}$ cellular: It will work!," IEEE Access, vol. 1, pp. 335-349, 2013.
[3] J. G. Andrews, S. Buzzi, C. Wan, S. V. Hanly, A. Lozano, A. C. K. Soong, and J. C. Zhang, "What will 5G be ?," IEEE Journal on Selected Areas in Commun., vol. 32, pp. 1065-1082, June 2014.

[4] Q. C. Li, Geng Wu, and T. S. Rappaport, "Channel model for millimeterwave communications based on geometry statistics," in Globecom Workshops (GC Wkshps), 2014, pp. 427-432, 8-12 Dec. 2014.

[5] M. K. Samimi and T. S. Rappaport, "3-D statistical channel models for millimeter-wave outdoor mobile broadband communications," in 2015 IEEE International Conference on Communications (ICC), June 2015.

[6] W. Roh et al, "Millimeter-wave beamforming as an enabling technology for 5G cellular communications: theoretical feasibility and prototype results," IEEE Communications Magazine, vol. 52, no. 2, pp. 106-113, Feb. 2014.

[7] S. Han, C.-L. I, Z. Xu, and C. Rowell, "Large-scale antenna systems with hybrid analog and digital beamforming for millimeter wave 5G," IEEE Commun. Mag., vol. 53, no. 1, pp. 186-194, Jan. 2015.

[8] O. E. Ayach, R. W. Heath, S. Abu-Surra, S. Rajagopal, and Z. Pi, "Low complexity precoding for large millimeter wave MIMO systems," in 2012 IEEE International Conference on Communications (ICC), pp. 3724-3729, 10-15 June 2012.

[9] A. Alkhateeb, O. El Ayach, G. Leus, and R. W. Heath Jr, "Hybrid precoding for millimeter wave cellular systems with partial channel knowledge," in Proc. of the Information Theory and Applications Workshop (ITA), San Diego, CA, Feb. 2013.

[10] A. Alkhateeb, O. El Ayach, G. Leus, and R. W. Heath Jr, "Channel estimation and hybrid precoding for millimeter wave cellular systems," IEEE Journal of Selected Topics in Signal Processing, vol. 8, no. 5, pp. 831-846, Oct. 2014.

[11] W. Ni, X. Dong, and W.-S. Lu, "Near-optimal hybrid processing for massive MIMO systems via matrix decomposition," available online: arXiv: 1504.03777.

[12] J. Singh and S. Ramakrishna, "On the feasibility of codebook-based beamforming in millimeter wave systems with multiple antenna arrays," IEEE Transactions on Wireless Communications, vol. 14, no. 5, pp. 2670-2683, May 2015.

[13] L. Dai, X. Gao, J. Quan, S. Han, and C.-L. I, "Near-optimal hybrid analog and digital precoding for downlink mmWave massive MIMO systems," in 2015 IEEE International Conference on Communications (ICC), pp. 1334-1339, 8-12 June 2015.

[14] V. Raghavan, S. Subramanian, J. Cezanne and A. Sampath, "Directional beamforming for millimeter-wave MIMO systems," in 2015 IEEE Global Communications Conference (GLOBECOM), pp. 1-7, 2015.

[15] R. Rajashekar and L. Hanzo, "Hybrid beamforming in mm-wave MIMO systems having a finite input alphabet," IEEE Transactions on Communications, available on IEEE early access.

[16] L. Liang, W. Xu and X. Dong, "Low-Complexity hybrid precoding in massive multiuser MIMO systems," IEEE Wireless Communications Letters, vol. 3, no. 6, pp. 653-656, Dec. 2014.

[17] R. A. Stirling-Gallacher and M. S. Rahman, "Multi-user MIMO strategies for a millimeter wave communication system using hybrid beamforming," in 2015 IEEE International Conference on Communications (ICC), pp. 2437-2443, 2015.

[18] J. Choi, "Beam selection in mm-Wave multiuser MIMO systems using compressive sensing," IEEE Transactions on Communications, vol. 63, no. 8, pp. 2936-2947, Aug. 2015 .

[19] A. Alkhateeb, G. Leus and R. W. Heath, "Limited feedback hybrid precoding for multi-user millimeter wave systems," IEEE Transactions on Wireless Communications, vol. 14, no. 11, pp. 6481-6494, Nov. 2015.

[20] W. Ni and X. Dong, "Hybrid block diagonalization for massive multiuser MIMO systems," IEEE Transactions on Communications, vol. 64, no. 1, pp. 201-211, Jan. 2016.

[21] T. E. Bogale and L. B. Le, "Beamforming for multiuser massive MIMO systems: Digital versus hybrid analog-digital," in Proc. IEEE Global Telecommun. Conf. (GLOBECOM), pp. 4066-4071, Dec. 2014.

[22] D. J. Love and R. W. Heath, "Equal gain transmission in multiple-input multiple-output wireless systems," in 2002 IEEE Global Telecommunications Conference GLOBECOM '02, vol.2, pp. 1124-1128, 2002.

[23] J. A. Tropp and A. C. Gilbert, "Signal recovery from random measurements via orthogonal matching pursuit," IEEE Transactions on Information Theory, vol. 53, no. 12, pp. 4655-4666, Dec. 2007.

[24] Lai-U Choi and R. D. Murch, "A transmit preprocessing technique for multiuser MIMO systems using a decomposition approach," in IEEE Transactions on Wireless Communications, vol. 3, no. 1, pp. 20-24, Jan. 2004.

[25] Q. H. Spencer, A. L. Swindlehurst and M. Haardt, "Zero-forcing methods for downlink spatial multiplexing in multiuser MIMO channels," in 
IEEE Transactions on Signal Processing, vol. 52, no. 2, pp. 461-471, Feb. 2004.

[26] D. Luenberger, "Optimization by vector space methods" John Wiley, 1997.

[27] Y. Fang, K. A. Loparo, and X. Feng, "Inequalities for the trace of matrix product," IEEE Transactions on Automatic Control, vol. 39, no. 12, pp. 2489-2490, Dec. 1994.

[28] G. H. Golub and C. F. Van Loan, "Matrix Computations," Johns Hopkins University Press, 1996.

[29] X. Zhang, A. F. Molisch and Sun-Yuan Kung, "Variable-phase-shiftbased RF-baseband codesign for MIMO antenna selection," IEEE Transactions on Signal Processing, vol. 53, no. 11, pp. 4091-4103, Nov. 2005.

[30] P. Sudarshan, N. B. Mehta, A. F. Molisch and J. Zhang, "Channel statistics-based RF pre-processing with antenna selection," IEEE Transactions on Wireless Communications, vol. 5, no. 12, pp. 3501-3511, Dec. 2006.

[31] D. Tse and P. Viswanath, "Fundamentals of wireless communication." Cambridge University Press, 2005.

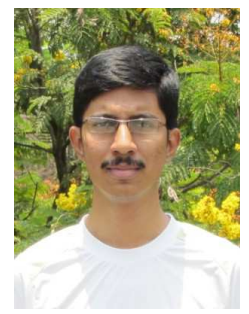

Rakshith Rajashekar received the B.E. degree in electrical communication engineering from Visvesvaraya Technological University, Karnataka, India, in 2007. He received his Ph.D.from the Department of Electrical Communication Engineering, Indian Institute of Science (IISc), India, in 2014. He is presently working as a Research Fellow at the University of Southampton (UoS), UK. Before joining the UoS, he worked at Accord Software \& Systems, Bangalore, India, as a Systems Engineer from 2007 to 2009, and as a Senior Scientist at Broadcom Communications from 2014 to 2015 . His present research interests include millimeter wave communication, visible light communication, with a focus on space-time signal processing and coding.

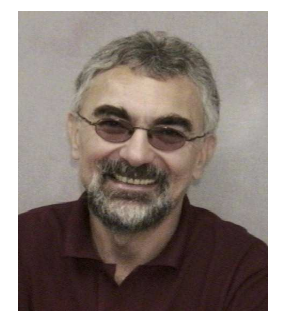

Lajos Hanzo (http://www-mobile.ecs.soton.ac.uk) FREng, FIEEE, FIET, Fellow of EURASIP, DSc received his degree in electronics in 1976 and his doctorate in 1983. In 2009 he was awarded the honorary doctorate "Doctor Honoris Causa" by the Technical University of Budapest. During his 40-year career in telecommunications he has held various research and academic posts in Hungary, Germany and the UK. Since 1986 he has been with the School of Electronics and Computer Science, University of Southampton, UK, where he holds the chair in telecommunications. He has successfully supervised $100+\mathrm{PhD}$ students, co-authored 18 John Wiley/IEEE Press books on mobile radio communications totalling in excess of 10000 pages, published $1650+$ research entries at IEEE Xplore, acted both as TPC and General Chair of IEEE conferences, presented keynote lectures and has been awarded a number of distinctions. Currently he is directing a 50-strong academic research team, working on a range of research projects in the field of wireless multimedia communications sponsored by industry, the Engineering and Physical Sciences Research Council (EPSRC) UK, the European IST Programme and the Mobile Virtual Centre of Excellence (VCE), UK. He is an enthusiastic supporter of industrial and academic liaison and he offers a range of industrial courses. He is also a Governor of the IEEE VTS. During 2008 - 2012 he was the Editor-inChief of the IEEE Press and a Chaired Professor also at Tsinghua University, Beijing. His research is funded by the European Research Council's Senior Research Fellow Grant. For further information on research in progress and associated publications please refer to http://www-mobile.ecs.soton.ac.uk Lajos has $30000+$ citations. 